\title{
ELECTRON SPIN RESONANCE (ESR) DATING IN KARST ENVIRONMENTS
}

\author{
DOLOČANJE STAROSTI V KRASU S POMOČJO ELEKTRONSKE \\ SPINSKE RESONANCE (ESR)
}

\author{
Bonnie A. B. BLACKWELL ${ }^{1}$
}

\begin{abstract}
UDC 543.4:551.44, 902.035:551.44

Bonnie A. B. Blackwell: Electron Spin Resonance (ESR) Dating in Karst Environments

Electron spin resonance (ESR) dating has been developed for many materials, including hydroxyapatite in enamel, bone, and some fish scales, aragonite and calcite in travertine, molluscs, and calcrete, and quartz from ash, which have many potential applications in karst settings. Although the complexity of the signals in some materials has hampered routine application, research is solving these problems to make the method even more widely applicable. When tested against other dating techniques, age agreement has usually been excellent. Generally, the most reliable applications seem to be tooth enamel, some mollusc species, calcite deposits, and quartz minerals. ESR dating uses signals resulting from trapped charges created by radiation in crystalline solids. Ages are calculated by comparing the accumulated dose in the dating sample with the internal and external radiation dose rates produced by natural radiation in and around the sample. For fossils and authigenic minerals, no zeroing is necessary to obtain accurate ages. In sediment which contains reworked mineral clasts, ESR can be used to date the age of the mineral grain itself if it was not zeroed during erosion. For dating the sedimentation age, however, ESR signals must have been zeroed in order to give the correct age. High pressure, heating, and in some minerals, light exposure and grinding can zero an ESR signal, but some like hydroxyapatite have very high stability at surface temperatures. For materials that absorb uranium (U) during their burial history, such as teeth, bones, or mollusc shells, the age calculation considers their $\mathrm{U}$ uptake by cross calibrating with $\mathrm{U}$ series or $\mathrm{U} / \mathrm{Pb}$ dating or by assuming different uptake models. Some difficulties in calculating the external dose rate can be overcome by applying the ESR isochron method, in which the sample acts as its own dosimeter. In open-air karst environments, changes in
\end{abstract}

Izvleček UDK 543.4:551.44, 902.035:551.44 Bonnie A. B. Blackwell: Določanje starosti v krasu s pomočjo elektronske spinske resonance (ESR)

Metoda ugotavljanja starosti s pomočjo elektronske spinske resonance (ESR), je bila razvita za najrazličnejše gradivo in snovi, vključno hidroksiapatit, emajl, kost, ribjo lusko, aragonit in kalcit v lehnjaku, školjčnih lupinah in kalcitnih skorjah, kremen v pepelu, kar vse nudi široke možnosti za uporabo v kraškem okolju. Čeprav pestrost signalov v nekaterem gradivu ovira vsestransko uporabnost, raziskave rešujejo te težavein tako je ta metoda še bolj vsestransko uporabna. Ob primerjanju $\mathrm{z}$ drugimi tehnikami datacije, je ujemanje $\mathrm{v}$ starosti običajno odlično. Na splošno je ta metoda najbolj zanesljiva, če se uporablja za zobno sklenino, nekatere vrste školjk, odkladnine kalcita in minerale kremena. ESR metoda izkorišča za datiranje signale, ki so posledica napetosti, ki jih ustvarja sevanje v kristalih. Starost se preračuna s pomočjo primerjave ohranjene količine sevanja $\mathrm{v}$ vzorcu za datiranje $\mathrm{z}$ deležem notranje in zunanje količine naravnega sevanja $\mathrm{v}$ vzorcu in okoli njega. Za fosile in avtigeno snov »ničenje« signalov za ugotavljanje prave starosti ni potrebno. Za sedimente, ki vsebujejo ponovno odložene mineralne skupke, se ESR lahko uporablja za določanje starosti samih mineralnih zrn, če tekom erozije niso bili signali "ničeni«. Za datiranje starosti sedimentacije pa morajo biti ESR signali »ničeni«, da dobimo pravilno starost. Visok pritisk, segrevanje in, v primeru nekaterih mineralov, izpostavljenost svetlobi ter drobljenje lahko »ničijo« signal ESR, medtem ko so nekateri drugi, npr. hidroksiapatit, pri površinski temperaturi zelo stabilni. Pri gradivu, ki v času, ko je pokopano v sedimentih, absorbira uran (U), kot so zobje, kosti, školjčne lupine, je treba pri ugotavljanju starosti upoštevati količino prejetega $U$ s pomočjo križnega umerjanja $\mathrm{U}$ vrste ali datiranja s pomočjo U/Pb oziroma upoštevati ustrezne modele. Težave pri računanju prejetega zunanjega sevanja je mogoče premostiti s pomočjo ESR izohrone metode, kjer je vzorec tudi svoj lastni dozimeter. V primeru površinskega kraškega okolja

${ }^{1}$ Department of Chemistry, Williams College, Williamstown, MA, 01267, USA

e-mail: bonnie.a.b.blackwell@williams.edu

Prejeto / Received: 29.03.2005 
the external dose rate due to altered sediment cover, and hence, changing cosmic dose rates, need to be modelled. For all karst environments, sedimentary water concentration and mineralogical variations with time also need to be considered. Many ESR applications are currently used in karst settings, but several more are also possible.

Key words: ESR (electron spin resonance) dating; ESR microscopy; cave geochronology; spring geochronology; teeth; mollusc shells; ratite eggshells; travertine; authigenic carbonates; authigenic salts; heated flint. je potrebno modleirati spremembe v količini sprejetega zunanjega sevanja zaradi sprememb $\mathrm{v}$ sedimentnem pokrovu, torej zaradi sprememb deleža kozmičnega sevanja. $\mathrm{V}$ vseh kraških okoljih pa je treba upoštevati količino vode v sedimentu ter sčasoma nastale mineraloške spremembe. Danes je ESR metoda uporabljana $\mathrm{v}$ številnih primerih na krasu, a so možnosti njene uporabe še večje. Ključne besede: ESR (elektronska spinska resonanca) datiranje, ESR mikroskopija, geokronologija jame, geokronologija izvira, zobje, školjčne lupine, lehnjak, avtigeni karbonati, avtigene soli, segrevan kremen.

\section{INTRODUCTION}

Electron spin resonance (ESR) dating can provide chronometric (absolute) dates over a substantial time range, from as young as $0.5 \mathrm{ka}$ to about 5-10 Ma, currently with $2-10 \%$ precision. ESR, like its sister methods, thermo(TL), optically stimulated (OSL), and radio- luminescence (RL), relies on detecting trapped charges induced by radiation in crystals. ESR can be used to date many materials that are commonly encountered at karst sites, as well as samples curated in museums, and new applications are constantly being added. ESR's importance in dating Quaternary and Pliocene sites has now been well demonstrated in archaeological contexts where it has dramatically changed our understanding of human origins and cultures (e.g., references in Blackwell, 2001). Many of these applications were in caves or abris, but could equally well be applied to open-air sites where the research questions are similar.

In karst settings, ESR provides several advantages over rival methods. For example, it can date fossils much older than the ${ }^{14} \mathrm{C}$ dating limit $(\sim 40-50 \mathrm{ka})$. ESR does not require a handy volcano to produce datable rocks like ${ }^{39} \mathrm{Ar} /{ }^{40} \mathrm{Ar}$ does, because ESR can also date fossils and sediment directly. Unlike the uranium (U) series methods, ESR can date most mollusc species accurately, as well as authigenic cements, some clays, and aeolian sediment. Unlike TL, OSL, and RL, ESR does not require that signals be completely zeroed for most applications and signals do not suffer anomalous fading. ESR's potential to date a wide variety of sample types will undoubtedly continue to make it an important research tool in late Cenozoic karst settings.

\section{BRIEF HISTORY OF ESR DATING}

In 1936, Gorter and colleagues delineated the basic principles of ESR spectroscopy. Early attempts to date many different materials were unsuccessful, despite ESR having been considered analogous to TL in its application. Finally, in 1975, Ikeya successfully dated a stalagmite from Akiyoshi Cave, Japan.

A flurry of research quickly followed in which geochronologists tried to date everything from fossils to dried blood, and quartz to engine oil, much of it led by Ikeya and other Japanese scientists. Important early applications included attempts to date fault gouge, burnt flint, teeth, and bones. Unfortunately, some early inaccurate applications to controversial archaeological sites, such as Caune de l'Arago, hampered its early acceptance by scientists. Currently, some 60 laboratories worldwide, 25 in Japan alone, research ESR dating and dosimetry, but only about 10 routinely perform dating. Its most common and reliable applications today include tooth enamel, molluscs, corals, and quartz from fault gouge, but research for food irradiation and retrospective dosimetry is producing numerous basic studies that may lead to new geological and paleontological uses. Developments in ESR imaging and microscopy promise many new mineralogical and paleontological applications.

\section{PRINCIPLES OF ESR ANALYSIS}

When minerals experience natural radiation, they gradually accumulate trapped unpaired electrons and positively charged "holes" (Figure 1a), which each produce characteristic ESR signals detectable with an ESR spectrometer.
Several such signals result from defects in the crystalline structure associated with trace contaminants. If the ESR signal height (intensity) for a radiation-sensitive signal can be converted into an accumulated dose (Figure 1b) 

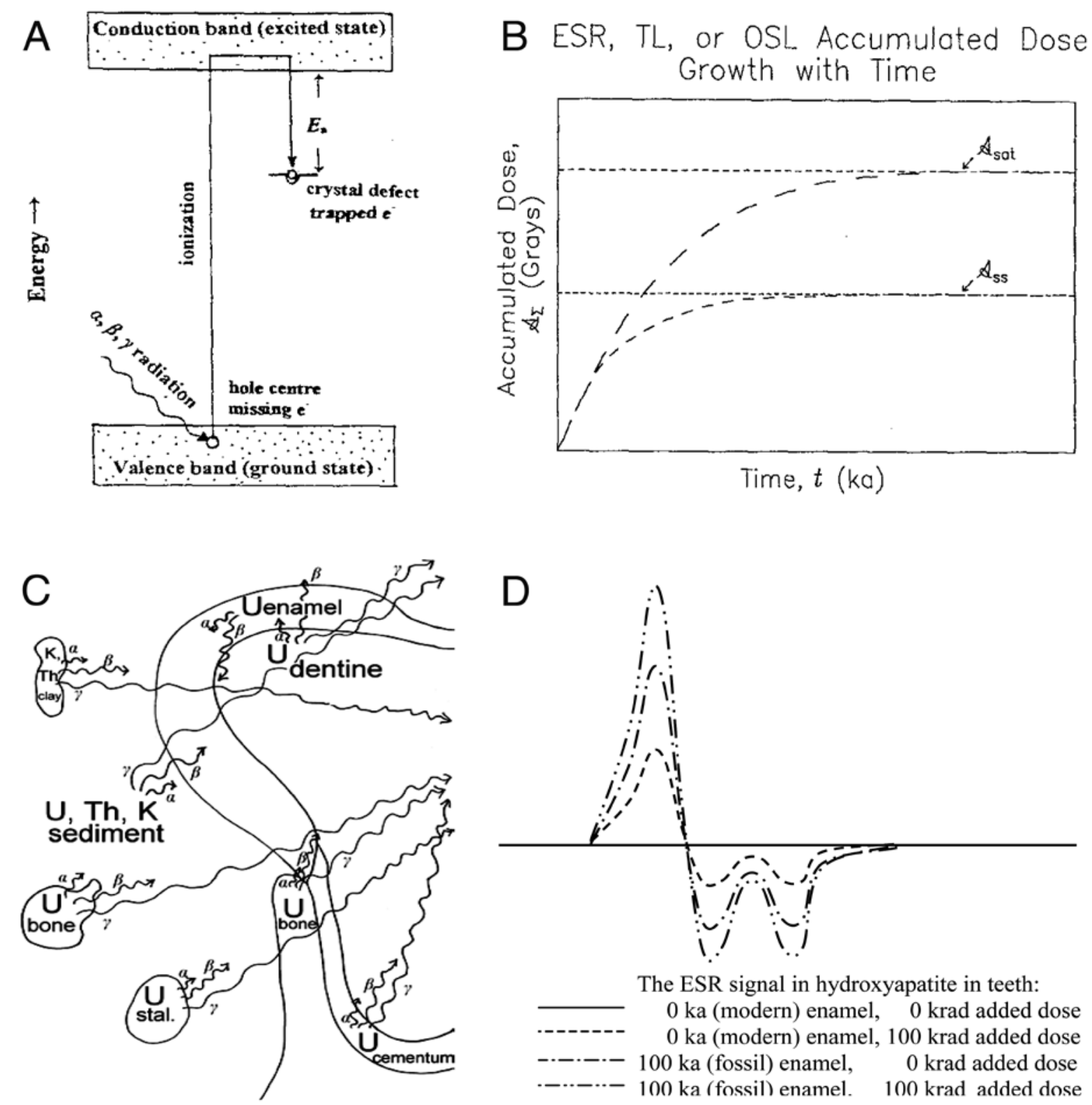

The ESR signal in hydroxyapatite in teeth:

- $0 \mathrm{ka}$ (modern) enamel, $0 \mathrm{krad}$ added dose

$0 \mathrm{ka}$ (modern) enamel, $100 \mathrm{krad}$ added dose

-.--.-- $100 \mathrm{ka}$ (fossil) enamel, $0 \mathrm{krad}$ added dose

-..- $100 \mathrm{ka}$ (fossil) enamel. $100 \mathrm{krad}$ added dose

Fig. 1: ESR signal production.

With increased irradiation, the ESR signal's intensity grows, eventually reaching saturation:

a. After absorbing energy from incident radiation, excited electrons move through the conduction band. Although most return to the ground state, a few become trapped in charge site defect (traps, often at trace elements substituents in the crystal lattice) that each have specific energies above the ground state. ESR signals result from the magnetic fields generated by such unpaired electrons and the empty holes they have left behind. With irradiation, such trapped electrons and charged holes, which each produce characteristic signals, gradually accumulate in the materials.

b. With natural irradiation, the signal saturates at its maximum (saturated) accumulated dose, $A_{\Sigma, \text { sat }}$ or at a lesser dose, a steady state accumulated dose, $A_{\Sigma, s}$, where signal fading loss equals signal production.

c. For any sample, many possible radiation sources may exist to produce the ESR signal. In addition to the $U$ in the enamel itself, the dentine and other tissues in the tooth are emitting radiation, as are all the components in the sediment within $30 \mathrm{~cm}$ of the tooth.

d. In most fresh teeth, the hydroxyapatite signal has zero intensity. The exceptions are teeth that have experienced a nuclear accident. If a fresh tooth experiences irradiation, a measureable signal will appear after $\sim 0.01$ Grays exposure, making it a useful signal for monitoring dose exposure during nuclear accidents. In a fossil tooth, a measureable signal is present after $\sim 1-20 \mathrm{ka}$, depending on the total dose rate that the tooth is experiencing. When any tooth experiences artificial irradiation, the signal will grow larger. 
and the radiation dose rate experienced by the sample during its deposition is known or can be modelled (Figure 1c), a date can be calculated. ESR dates can be obtained using any material, which has a radiation-sensitive ESR signal (e.g., Figure 1d), provided it satisfies the following criteria,

1. At the time of interest, the mineral's ESR signal was initially, or was reset to, 0.0 .

2 . The signal lifetime, $\tau$, exceeds the site age by at least two orders of magnitude.

3. The accumulated dose, $A_{\Sigma}$, is less than the saturation level in the material.

In karst contexts, tooth enamel, clean carbonates (speleothem, travertine, mollusc shells, calcareous cements, calcrete), and heated or bleached siliceous rock (flint or quartz) have several applications. Many salts may eventually produce valid dates, but the techniques have not been perfected yet. Sediment dates have been attempted, but problems related to incomplete zeroing must still be resolved. This discussion will focus on karst applications, illustrated where possible by karst examples. It omits other applications, although other recent reviews (e.g., Blackwell, 2001; Falguères, 2003) do discuss other applications.

A few technical terms become essential here. An ESR spectrometer uses a microwave signal to create resonance between the unpaired electrons in minerals and an externally applied strong magnetic field. Lande's factor, called the g value, is a dimensionless number that uniquely describes the ESR characteristics for any peak. Pulsed X-, K-, or Q-band ESR may ultimately improve our ability to separate interference signals (e.g., Grün et al., 1997; Kinoshita et al., 2004). Although other bands, such as Q- or L-band, are occasionally used to examine signals in more detail, for most ESR dating, spectra are analyzed in the X-band at 1-10 $\mathrm{mW}$ power using microwave frequencies near $8-10 \mathrm{GHz}$ under a $100 \mathrm{kHz}$ field modulation. Under these conditions, most geologically or archaeologically interesting ESR signals fall within $3>$ g > 1.9 (Blackwell, 1995, Table 2).

Zeroing reduces an ESR signal's intensity to a level indistinguishable from background levels. Most newly formed minerals have no measurable ESR signals. In a mineral with an accumulated dose (i.e., a measurable signal; $\left.A_{\Sigma}>0\right)$, several physical processes can also zero a signal. Strong heating to temperatures above $250-500^{\circ} \mathrm{C}$, depending on the mineral, will also zero most ESR signals (Figure 2b). For some signals in a few minerals, exposure to intense sunlight can zero (bleach) the signal (Figure 2a). Luckily, for the radiation-sensitive signals in most minerals, sunlight causes little or no signal loss. High

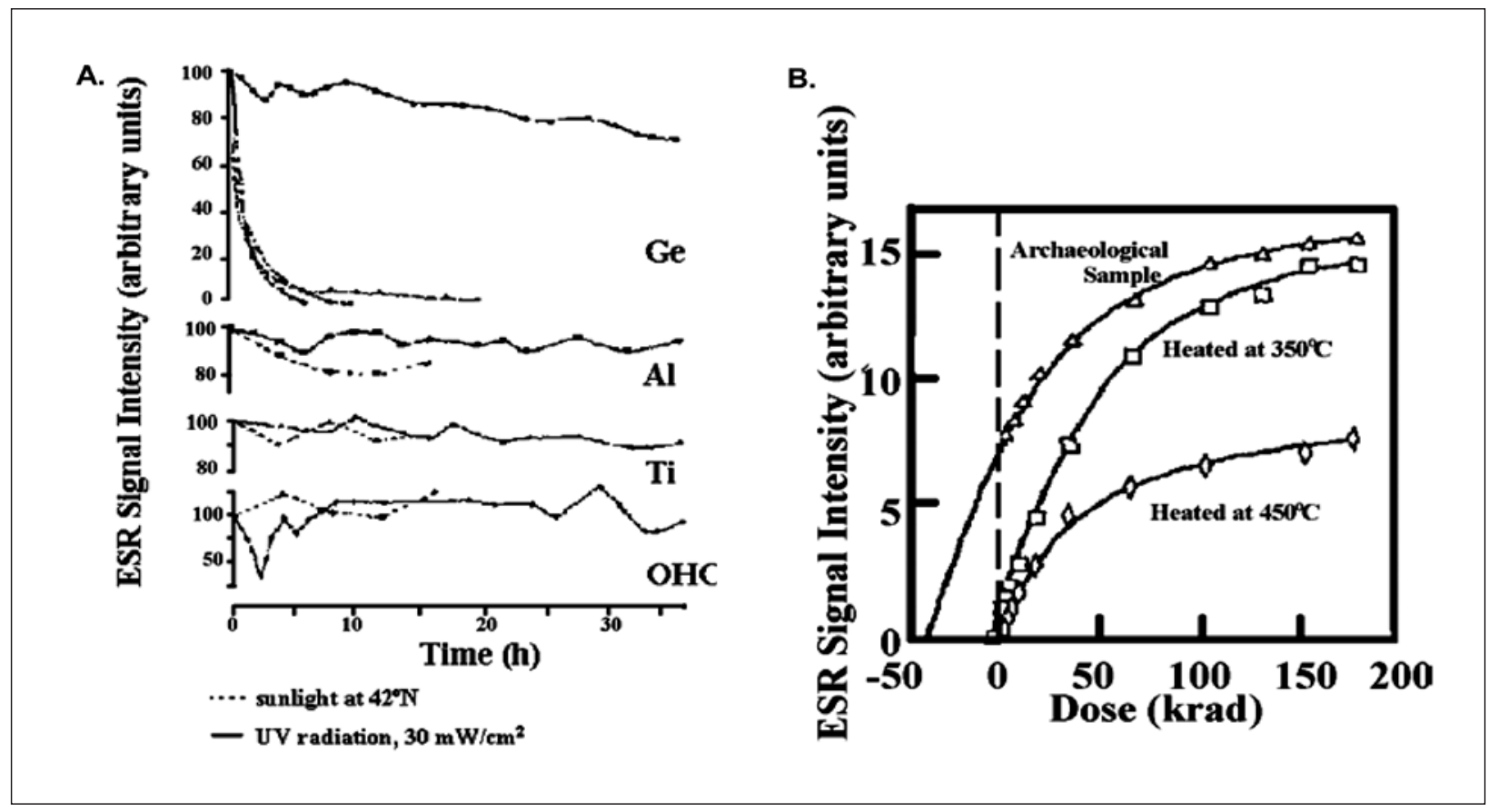

Fig. 2: Zeroing in quartz and chert. In quartz, several signals can be zeroed using different techniques:

a. Exposure to intense UV radiation and sunlight can completely bleach the Ge (germanium) signal and partially bleach the Al (aluminium), Ti (titanium), and OHC (oxygen hole) signals.

b. Heating archaeological chert to high temperature can zero the $E^{\prime}$ signal, reducing its accumulated dose, $A_{\Sigma}$, to 0 . After zeroing, the signals can regrow if given more irradiation.

(adapted from Blackwell, 2001). 

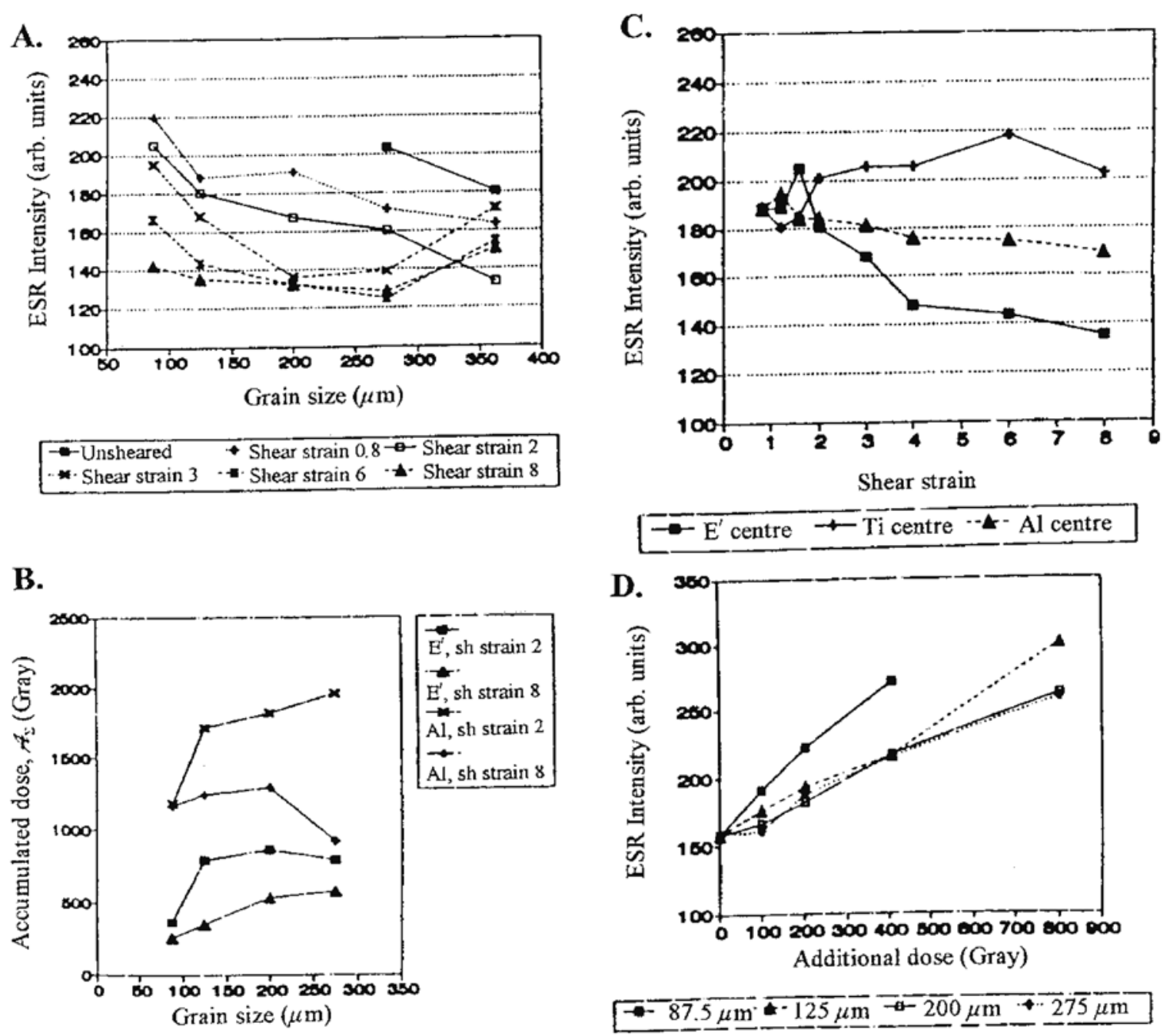

$-87.5 \mu \mathrm{m}-125 \mu \mathrm{m}=200 \mu \mathrm{m}-27 \overline{\mathrm{m}}$

Fig. 3: Effects from shear strain on ESR signals in quartz. Shear strain will reset most ESR signals:

a. As strain increases, the differences in ESR intensity between different grain size fractions decreases.

b. At a normal stress of $10 \mathrm{MPa}$, the measured accumulated (equivalent) dose, $A_{\Sigma}{ }^{\prime}$, decreases with decreasing grain size for both the $E^{\prime}$ and Al signals, until at a small grain size the two signals give equal $A_{\Sigma}$, determinations.

c. While the E' signal is the most easily reset, strain also affects the Al signal. The Ti signal appears unaffected.

d. During artificial irradiation for producing a growth curve, the smaller grain sizes show the greatest sensitivity and the most well behaved growth curves.

(modified from Lee \& Schwarcz, 1993).

pressure or strain that builds up in faults can partially or fully reset some signals, as can the strain developed during comminution during an earthquake or grinding for sample preparation (Figure 3). Remineralization and diagenesis add new minerals whose radiation-sensitive signals will be zero at formation. Therefore, if the original and new minerals have signals with similar g values, the resultant complex signal may be impossible to resolve, adding inaccuracies to the age determination. If, howev- er, the new signals do not interfere with the original signals, as is true for tooth enamel, only the dating signal's intensity is reduced, thereby reducing the discriminatory range and dating limits for the technique (Skinner et al., 2000).

The method's reliability depends on the signal's thermal stability. Signals which zero easily at typical Earth surface temperatures have little value for dating, but may provide other information. The mean signal lifetime, $\tau$, 
must exceed the desired dating range by at least 2-3 orders of magnitude to ensure reliable ages. In tooth enamel, for example, $\tau \approx 10^{19} \mathrm{y}$ (Skinner et al., 2000), sufficiently long, in theory at least, to date anything within the history of the universe. Unlike TL, no datable ESR signal appears to suffer anomalous fading. In practice, however, most signals have a finite saturation limit, beyond which no new trapped electrons are formed. Many minerals also have a steady state level, somewhat lower than their saturation level, caused by electron loss and retrapping (Figure $1 \mathrm{~b}$ ). The mean signal lifetime and the steady state limit or saturation limit define the maximum datable age, while the ESR spectrometer's ability to discriminate between the dating signal and its surrounding background determines the minimum dating limit. Both limits differ depending on the mineral and its habit in the material to be dated. The radiation dose rates experienced by the sample determine how those limits are translated into an actual age. If samples experience high radiation dose rates, the minimum datable age will be relative low, but so will its maximum datable age, and conversely, low radiation dose rates mean higher minimum and maximum limits.

The ESR signal height (Figure 1d) is proportional to the number of trapped charges at that lattice site, and, therefore, to the total radiation dose, $A_{\Sigma}$, that the material has experienced. The ESR age, $t 1$, the time that has elapsed since the mineral formed and began to accumulate charges then is calculated from Equation 1,

$\mathrm{A}_{\Sigma}=\mathrm{A}_{\text {int }}+\mathrm{A}_{\text {ext }}=\int_{\text {to }}^{\mathrm{t} 1} \mathrm{D}_{\Sigma}(\mathrm{t}) \mathrm{dt}=\int_{\text {to }}^{\mathrm{t1}}\left(\mathrm{D}_{\text {int }}(\mathrm{t})+\mathrm{D}_{\text {ext }}(\mathrm{t})\right) \mathrm{dt}$

where

$\mathrm{A}_{\Sigma}=$ the total accumulated dose in the sample,

$\mathrm{A}_{\mathrm{int}}=$ the internally derived accumulated dose component,

$\mathrm{A}_{\text {ext }}=$ the externally derived accumulated dose component,

$\mathrm{D}_{\Sigma}(\mathrm{t})=$ the total dose rate

$\mathrm{D}_{\text {int }}(\mathrm{t})=$ the total dose rate from internal sources: $\mathrm{U}$, its daughters, and any other radioisotopes,

$\mathrm{D}_{\text {ext }}(\mathrm{t})=$ the total dose rate from the external environment: sedimentary $\mathrm{U}$, Th, and $\mathrm{K}$, and cosmic dose,

$\mathrm{t}_{1}=$ the sample's age,

$\mathrm{t}_{0}=$ today.

For samples in which the total dose rate, $\mathrm{D}_{\Sigma}(\mathrm{t})$, is constant, this reduces to

$$
\mathrm{t}_{1}=\frac{\mathrm{A}_{\Sigma}}{\mathrm{D}_{\Sigma}(\mathrm{t})}
$$

\section{SAMPLE COLLECTION}

An ideal ESR sample should be as pristine as possible. To improve precision and accuracy, both the dating sample and any associated sediment samples should not experience the following treatments during or after excavation:

1. Glues, shellacs, and other preservatives can add contaminant $U$ to the sample that reduces the accuracy of internal dose rate measurements, as well as organic compounds that might cause ESR signal interference.

2. Washing may remove $U$, datable mineral, and sediment. Sediment attached to the sample may offer the only chance to measure the external dose rates.

3. If used to remove samples from cemented sediment, acid dissolution can dissolve the sample and leach its $U$.

4. Removing attached bone from teeth reduces the accuracy of the external dose rate measurements.

5. Removing attached sediment from any sample reduces the accuracy of the external dose rate measurements.

6. Sample numbering uses inks and paints that can add contaminant organic compounds if applied to the sample.

7. Allowing clay samples to dry necessitates extensive grinding during preparation which can partially bleach some ESR signals.
8. Packing samples for transport with materials, such as old newspapers, dyed paper, etc., can cause trace elements or organic contamination if they contact the sample. The best packing is cheap unbleached, unperfumed toilet paper.

Although preservatives, if available, can be analyzed to correct for contamination effects, any resulting age will still have reduced precision. Fossils can be cast, providing that the casting resin and powder have been tested for contamination potential first.

For all dating samples except teeth, diagenesis or signal interference may cause some samples to be unsuitable (Table 1). Since fossils can be easily reworked into younger depositional units, any sampling program should collect at least 8-10 samples from each stratigraphic unit to increase the chance that the samples analyzed provide dates related to the event of interest. Although the required sample weight varies depending on the auxiliary analyses necessary (Table 1), the ESR analysis itself, and the associated NAA or geochemical analyses to measure the internal dose rate, require 1-2 g of pristine datable mineral per standard ESR subsample. For some materials, especially those prone to diagenesis, it is necessary to check for secondary mineralization and remineraliza- 
tion, which affect ESR signal intensities (Table 1), thus requiring larger samples. For samples needing to be separated into discrete mineral phases, such as authigenic cements, caliche, calcrete, and gypcrete, the pristine mineral must be separated from the adjacent sediment, often necessitating much larger samples (usually, at least 15-20 g). For ESR dating sediment, pristine sample blocks of $\sim 0.5 \mathrm{~kg}$ cut from thick or extensive units provide the best results, if available. For very small samples (100-200 $\mathrm{mg}$ ), the ramping irradiation technique can be used in which several aliquots are reirradiated several times, but the special handling does lengthen the total analysis time significantly.
Most curated museum samples require isochron analysis (see below), because sediment has not usually been preserved. Salt samples need to be stabilized to prevent remineralization or recrystallization during transport, as can occur with some hydrated salts. For samples intended for isochron analysis, samples should be photographed before shipping to ensure that broken samples can be reconstructed to maximize the number of viable subsamples. Samples should be packed tightly with minimal air to reduce sample breakage and bag destruction.

\section{SEDIMENT DOSIMETRY AND ASSOCIATED SEDIMENT SAMPLES}

Many karst sites have sediment which is inhomogeneous (i.e., "lumpy"; Figure 1d, 4d) for radioactive dose generation. This is particularly true in caves where sedimentary inhomogeneity is the rule, rather than the exception. Whenever possible, the external dose rates should be assessed using at least two procedures from among isochron analysis, sediment geochemistry, in situ $\gamma$ or TL dosimetry. For TL or $\gamma$ dosimetry, if dosimetry cannot be completed before collection, sampling locations need to be marked and preserved to permit future dosimetry. Effective TL dosimetry requires that the area within $3 \mathrm{~m}$ of the dosimeter insertion site be unaffected by further excavation or erosion for 6-12 months. In open-air sites, however, either $\gamma$ dosimetry or sedimentary analysis is preferred over TL dosimetry, because TL dosimeters rarely survive undisturbed for the needed time. Isochron analysis is still experimental for many materials.

With sedimentary geochemistry, the external dose field can be mathematically modelled reasonably accurately. In sediment, $\beta$ particles can penetrate about 2-3 $\mathrm{mm}$, and $\gamma$ radiation $\sim 30 \mathrm{~cm}$ (Figure 4). The sediment immediately attached to, or surrounding, the dating sample usually provides the only direct measurement for calculating the $\beta$ radiation dose rate. When using $\gamma$ or TL dosimetry, this sediment must still be analyzed geochemically to provide the external $\beta$ dose rate. Several sediment samples may be needed to represent the sphere influenced by $\gamma$ radiation $30 \mathrm{~cm}$ in radius around the dating sample.

Sediment sampling protocols vary with the bed or unit thickness, its mineralogy, and its grain size (see Table 2; Figure 5). In many sedimentary contexts, the radioactive element concentrations can vary dramatically over short distances if the sediment contains large clasts of several different minerals ("lumpy"; Figure 4d). This requires collecting several samples from each unit or bed which might have contributed to the dating sample's external dose rate. If the sediment contains a homogeneous grain mixture of fine to medium grained clasts, $\sim 5-10 \mathrm{~g}$ are sufficient for each associated sediment sample. For coarser sediment types, sediment samples should include representative portions of cobbles mixed with the matrix. Alternatively, separate matrix and cobble samples can be submitted, provided relative volume percentages of the various types are known. In units with fossils or artefacts, these must be considered as radioactive sources and analyzed also (Blackwell \& Blickstein, 2000). Generally, the larger the grains, the larger the sediment mass that will be needed. In well cemented sedimentary units (e.g., "breccias", etc.), a block of sediment ( $20 \mathrm{~cm}$ on a side) showing all representative grains, matrix, and cements on the surfaces often provides the best sample.

If all the sediment samples preferred in the ideal circumstance are not available, sediment from the same or similar beds as close as possible to the dating sample can still be used to assess the radiation dose field's variability and estimate external dose rates. For museum samples, any samples from nearby outcrops may provide valuable clues. Accurately recording and photographing each sediment sample relative to the dating sample ensures accuracy in modelling the external dose field. All in situ sediment samples should be placed in clean, sealed jars or doubly bagged in new zip-lock bags immediately after collection to retain sediment moisture for water concentration analysis. For sections that have been exposed for a long time, or archived sediment, sediment moisture content is not analyzed. 


\begin{tabular}{|c|c|c|c|c|c|c|c|c|c|c|c|c|c|c|c|c|c|c|}
\hline 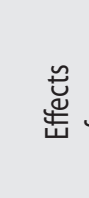 & ع & 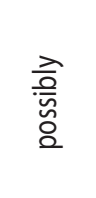 & 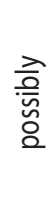 & $\begin{array}{l}\text { 츨 } \\
\text { 을 }\end{array}$ & $\begin{array}{l}\text { 츨 } \\
\text { 을 }\end{array}$ & $\begin{array}{l}\text { 츨 } \\
\text { 음 }\end{array}$ & $\begin{array}{l}\text { 츨 } \\
\text { 을 }\end{array}$ & 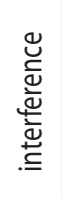 & 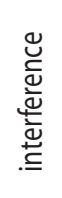 & 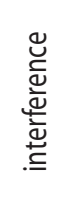 & 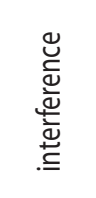 & 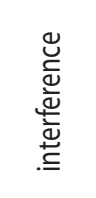 & 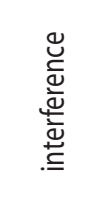 & 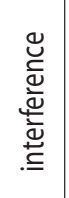 & 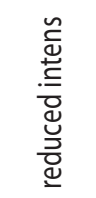 & 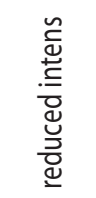 & 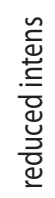 & 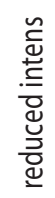 \\
\hline & 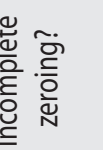 & $\stackrel{\tilde{\pi}}{\underline{x}}$ & $\stackrel{\pi}{\underline{s}}$ & $\stackrel{\pi}{\Omega}$ & $\stackrel{\pi}{\Omega}$ & $\stackrel{\pi}{\Omega}$ & $\stackrel{\tilde{\sigma}}{\Omega}$ & $\stackrel{\pi}{\Omega}$ & $\stackrel{\pi}{\Omega}$ & $\stackrel{\tilde{\Omega}}{\underline{x}}$ & $\stackrel{\pi}{\Omega}$ & $\stackrel{\frac{\pi}{c}}{\Omega}$ & 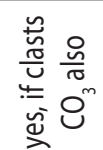 & $\stackrel{\pi}{\leq}$ & $\stackrel{\pi}{\Omega}$ & $\stackrel{\pi}{c}$ & 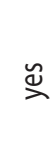 & 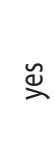 \\
\hline 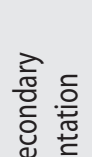 & 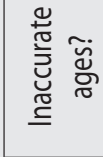 & 읃 & $\stackrel{\circ}{\complement}$ & 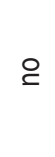 & 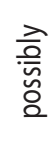 & 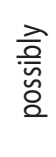 & ؟ & 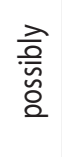 & 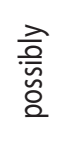 & 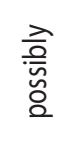 & 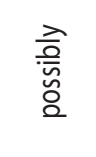 & 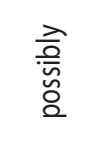 & 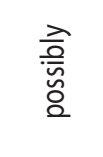 & 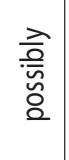 & 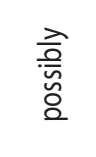 & 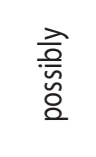 & 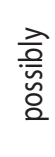 & 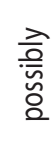 \\
\hline 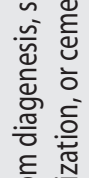 & 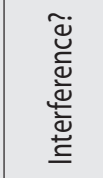 & $\frac{\overrightarrow{\mathrm{d}}}{\stackrel{\vec{\omega}}{\underline{\omega}}}$ & 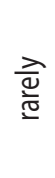 & 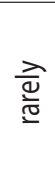 & 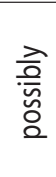 & $\begin{array}{l}\text { 츰 } \\
\stackrel{\overline{\check{\alpha}}}{\alpha}\end{array}$ & 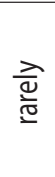 & $\begin{array}{l}\text { बे } \\
\underline{\underline{\underline{\underline{y}}}}\end{array}$ & $\begin{array}{l}\text { बे } \\
\underline{\underline{\underline{\underline{u}}}}\end{array}$ & $\begin{array}{l}\overrightarrow{\bar{\vartheta}} \\
\stackrel{\underline{\underline{\underline{y}}}}{ }\end{array}$ & 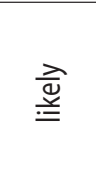 & $\begin{array}{l}\overrightarrow{\hat{Q}} \\
\underline{\underline{\underline{u}}}\end{array}$ & $\begin{array}{l}\overrightarrow{\bar{Q}} \\
\stackrel{\underline{\underline{\Xi}}}{ }\end{array}$ & $\begin{array}{l}\overrightarrow{\bar{Q}} \\
\underline{\underline{\underline{u}}}\end{array}$ & 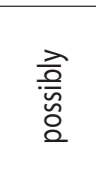 & $\begin{array}{l}\text { 츰 } \\
\overline{0} \\
\text { o }\end{array}$ & 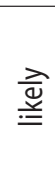 & 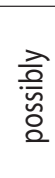 \\
\hline 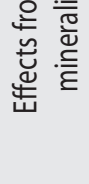 & 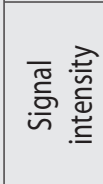 & 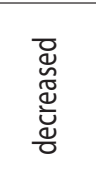 & 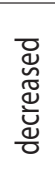 & 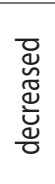 & 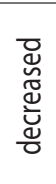 & 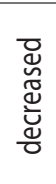 & 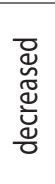 & 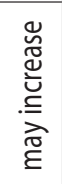 & 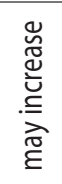 & 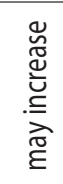 & 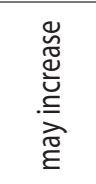 & 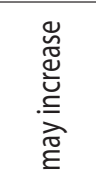 & 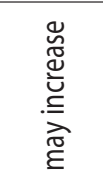 & 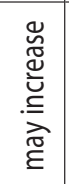 & 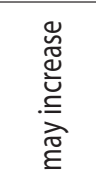 & 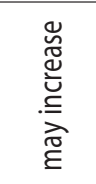 & 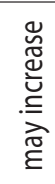 & 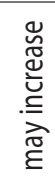 \\
\hline 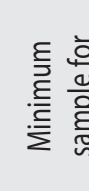 & 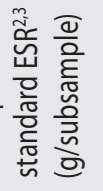 & $\stackrel{+}{m}$ & 음 & $\frac{5}{\sigma}$ & 유 & 윰 & $\stackrel{\substack{4 \\
\sim}}{\sim}$ & $\stackrel{\circ}{\stackrel{\circ}{\prime \prime}}$ & 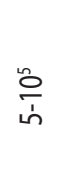 & 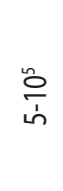 & 菅 & 㐫 & $\frac{\infty}{\grave{0}}$ & $\begin{array}{l}{ }^{+} \\
\stackrel{5}{\xi} \\
\stackrel{0}{0}\end{array}$ & 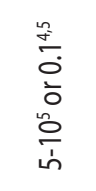 & 㐫 & 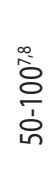 & $\stackrel{\infty}{\text { ํ่ }}$ \\
\hline & $\frac{\tilde{\ddot{m}}}{\stackrel{\tilde{u}}{\tilde{u}}}$ & 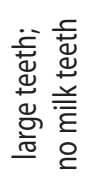 & 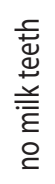 & 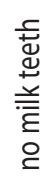 & 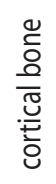 & 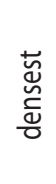 & $\begin{array}{l}\overline{\bar{\sigma}} \\
\text { त्र } \\
\text { ठ̀ }\end{array}$ & 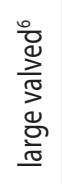 & 站 & 竎 & 竎 & 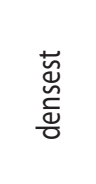 & 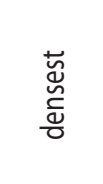 & 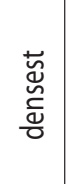 & 点 & 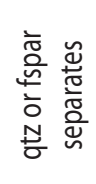 & 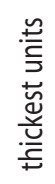 & 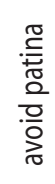 \\
\hline & 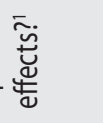 & ๕ & $\stackrel{\circ}{\complement}$ & ㅇ & ㅇ & ㅇ & 站 & 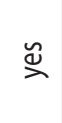 & 站 & 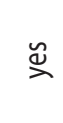 & 点 & $\stackrel{\mathscr{\pi}}{\mathrm{L}}$ & $\stackrel{\pi}{\complement}$ & $\stackrel{\pi}{\check{c}}$ & $\frac{\check{5}}{5}$ & $\stackrel{\pi}{\complement}$ & $\stackrel{\pi}{\complement}$ & $\stackrel{\stackrel{2}{\Omega}}{\Omega}$ \\
\hline & $\frac{\overline{\frac{\partial}{O}}}{\frac{0}{\bar{O}}}$ & $\stackrel{\beth}{\beth}$ & 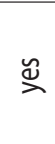 & ㅇ & 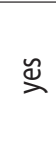 & $\stackrel{\check{\nu}}{\curvearrowright}$ & ๑ & 竎 & 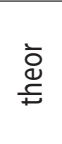 & 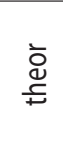 & 竎 & $\breve{\circlearrowright}$ & $\frac{\check{5}}{\leftrightarrows}$ & $\frac{\text { 妾 }}{5}$ & 站 & $\stackrel{\breve{\Perp}}{\curvearrowright}$ & $\stackrel{d}{\stackrel{d}{\rightleftarrows}}$ & 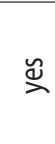 \\
\hline & 응 & $\stackrel{\circ}{\subseteq}$ & ๕ & 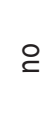 & ○ & ○ & ؟ & உ & உ & ๕ & $\stackrel{\circ}{\subseteq}$ & $\stackrel{\circ}{\subseteq}$ & $\cong$ & $\stackrel{\circ}{\subseteq}$ & ○ & 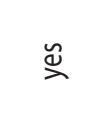 & $\stackrel{\coprod}{\beth}$ & 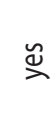 \\
\hline & $\frac{\pi}{\pi}$ & 这 & 齐 & 咅 & 这 & 这 & 全 & 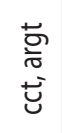 & ت্ర & 管 & 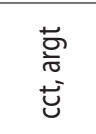 & 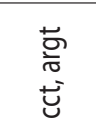 & ت્ర & 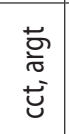 & $\frac{\pi}{2}$ & 要产 & N & $\frac{N}{\sigma}$ \\
\hline & ๕ & 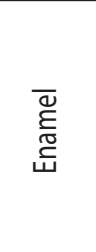 & & 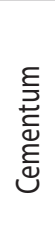 & ֻั & 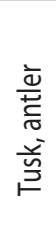 & 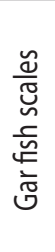 & 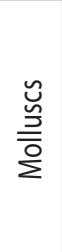 & 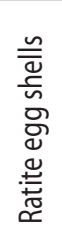 & 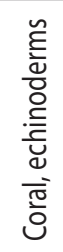 & 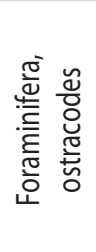 & 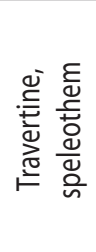 & 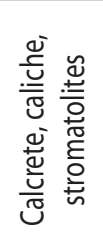 & 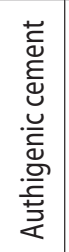 & 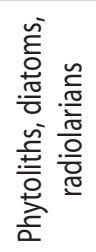 & 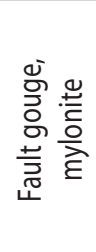 & $\frac{\frac{n}{2}}{\frac{2}{2}}$ & 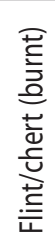 \\
\hline
\end{tabular}

Table 1: ESR Sample Types 


\begin{tabular}{|c|c|c|c|c|c|c|c|c|}
\hline 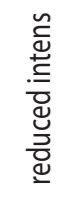 & 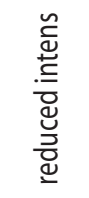 & 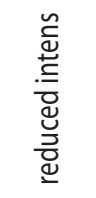 & 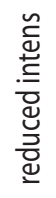 & $\frac{\sqrt{\frac{5}{5}}}{\mathrm{~J}}$ & 趌 & 胥 & 站 & $\frac{\sqrt{5}}{5}$ \\
\hline 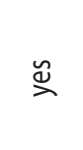 & 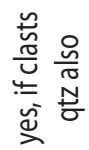 & $\stackrel{\dddot{\nu}}{\searrow}$ & $\stackrel{\pi}{\Omega}$ & $\stackrel{\tilde{\pi}}{\underline{x}}$ & $\stackrel{\pi}{\leq}$ & $\stackrel{\pi}{\Sigma}$ & $\stackrel{\tilde{\sigma}}{\mathrm{I}}$ & $\stackrel{\widetilde{\pi}}{\Omega}$ \\
\hline 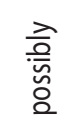 & $\begin{array}{l}\text { 층 } \\
\text { 뜽 } \\
\text { ¿ }\end{array}$ & $\begin{array}{l}\text { 층 } \\
\text { 응 }\end{array}$ & 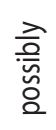 & 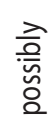 & $\begin{array}{l}\text { 츨 } \\
\text { 을 }\end{array}$ & 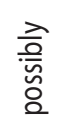 & $\begin{array}{l}\text { 층 } \\
\text { 을 }\end{array}$ & $\begin{array}{l}\text { 층 } \\
\text { 응 } \\
\text { a }\end{array}$ \\
\hline 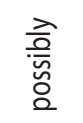 & 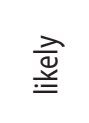 & 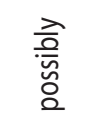 & 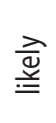 & 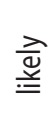 & 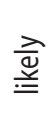 & 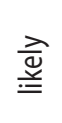 & $\underset{\underline{\vec{Q}}}{\stackrel{\overrightarrow{\underline{a}}}{ }}$ & 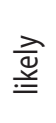 \\
\hline 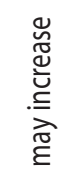 & 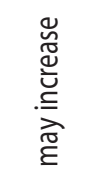 & 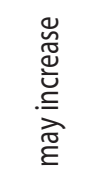 & 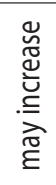 & 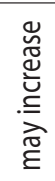 & 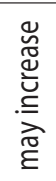 & 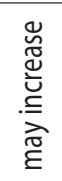 & 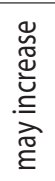 & 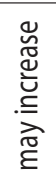 \\
\hline 离 & 文 & 文 & $\frac{5}{\circ}$ & $\frac{\infty}{\stackrel{0}{0}}$ & $\frac{t}{0}$ & 㐫 & 容 & 亩 \\
\hline 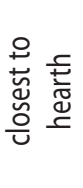 & 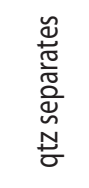 & 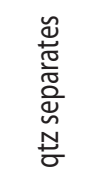 & 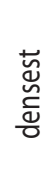 & 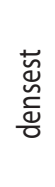 & 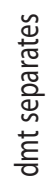 & 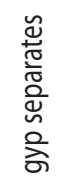 & 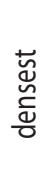 & 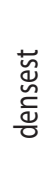 \\
\hline$\stackrel{\pi}{\leq}$ & $\stackrel{\pi}{\Omega}$ & $\stackrel{\tilde{\sigma}}{\mathrm{I}}$ & $\stackrel{\pi}{\check{L}}$ & $\stackrel{\pi}{\underline{\Sigma}}$ & $\stackrel{\pi}{\leq}$ & $\stackrel{\pi}{\underline{\Sigma}}$ & $\stackrel{\pi}{\leq}$ & $\stackrel{\pi}{\Omega}$ \\
\hline 站 & $\frac{\subsetneq}{5}$ & $\frac{\text { s }}{5}$ & 竎 & 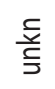 & $\frac{\frac{F}{5}}{5}$ & $\frac{\frac{F}{5}}{\leftrightarrows}$ & 站 & $\frac{5}{5}$ \\
\hline 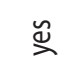 & 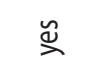 & 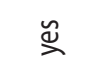 & & உ & 을 & ㅇ & 을 & 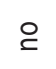 \\
\hline$\frac{N}{0}$ & $\frac{N}{\sigma}$ & 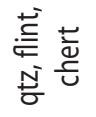 & $\frac{N}{0}$ & 苛 & 荎 & 츳 & $\underset{\frac{\mathbb{d}}{c}}{\vec{c}}$ & $\overline{\widetilde{\Phi}}$ \\
\hline 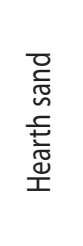 & 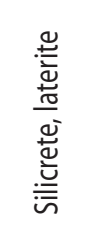 & 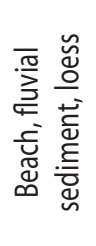 & 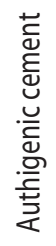 & 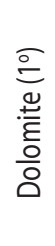 & 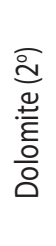 & 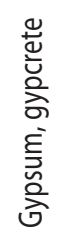 & 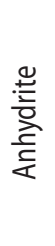 & $\frac{\stackrel{.5}{\overparen{T}}}{\frac{\pi}{I}}$ \\
\hline
\end{tabular}

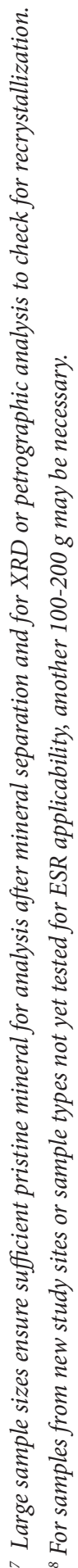




\begin{tabular}{|c|c|c|c|c|c|c|c|c|c|c|}
\hline \multicolumn{3}{|c|}{ Sedimentary Unit or Site ${ }^{1}$} & \multicolumn{2}{|c|}{ Sediment Grains (Clasts) } & \multirow{2}{*}{$\begin{array}{l}1^{0} \text { Dosing } \\
\text { Unit(s })^{4} \\
\text { Thickness } \\
(\mathrm{cm})\end{array}$} & \multicolumn{3}{|c|}{ Whole (Bulk) Sediment Samples } & \multirow{2}{*}{$\begin{array}{c}\text { Samples } \\
\text { of Clasts } \\
>0.5 \mathrm{~cm} \text { in } \\
\text { Diameter }^{6}\end{array}$} & \multirow[b]{2}{*}{ Fig. } \\
\hline Character' & $\begin{array}{l}\text { e.g. } \\
\text { Fig. }\end{array}$ & Type $^{1}$ & $\begin{array}{c}\text { Mineral } \\
\text { Compositions }^{2}\end{array}$ & $\begin{array}{l}\text { Grain } \\
\text { Size } \\
\text { Range }^{3}\end{array}$ & & $\begin{array}{l}\text { Mass }^{5} \\
\text { (g) }\end{array}$ & $\begin{array}{l}\text { from } 1^{0} \\
\text { Dosing } \\
\text { Unit(s) }\end{array}$ & $\begin{array}{l}\text { from } 2^{\circ} \\
\text { Dosing } \\
\text { Units }^{4}\end{array}$ & & \\
\hline $\begin{array}{l}\text { "Smooth", thickly } \\
\text { bedded sites }\end{array}$ & $4 c^{7}$ & $\begin{array}{l}\text { Thick } \\
\text { smooth }\end{array}$ & Homogeneous & Uniform & $>65$ & $5-10$ & $\begin{array}{l}\text { 4-6 orthogonally } \\
\text { oriented }\end{array}$ & none & $\begin{array}{l}\text { 1-3 for gravel- } \\
\text { sized matrix only }\end{array}$ & $5 a^{7}$ \\
\hline $\begin{array}{l}\text { "Smooth", thinly } \\
\text { bedded sites }\end{array}$ & $4 b^{7}$ & $\begin{array}{l}\text { Thin } \\
\text { smooth }\end{array}$ & Homogeneous & Uniform & $<65$ & $5-10$ & $\begin{array}{c}4-6 \text { orthogonally } \\
\text { oriented }\end{array}$ & $\begin{array}{l}\text { 3-5 for each unit } \\
\leq 30 \mathrm{~cm} \text { from } \\
\text { dating sample }\end{array}$ & $\begin{array}{l}\text { 1-3 for gravel- } \\
\text { sized matrix only }\end{array}$ & $5 b^{7}$ \\
\hline $\begin{array}{l}\text { "Lumpy", thickly } \\
\text { bedded sites }\end{array}$ & $4 d^{7}$ & $\begin{array}{l}\text { Thick } \\
\text { lumpy } 1\end{array}$ & Homogeneous & Non-uniform & $>65$ & $100-1000$ & $\begin{array}{c}4-6 \text { orthogonally } \\
\text { oriented }\end{array}$ & none & 1-3 per unit & $5 a^{7}$ \\
\hline $\begin{array}{l}\text { "Lumpy", thinly } \\
\text { bedded sites }\end{array}$ & $4 d^{7}$ & $\begin{array}{c}\text { Thin } \\
\text { lumpy } 1\end{array}$ & Homogeneous & Non-uniform & $<65$ & $100-1000$ & $\begin{array}{c}4-6 \text { orthogonally } \\
\text { oriented }\end{array}$ & $\begin{array}{l}3-5 \text { for each unit } \\
\leq 30 \mathrm{~cm} \text { from } \\
\text { dating sample }\end{array}$ & 1-3 per unit & $5 b^{7}$ \\
\hline $\begin{array}{l}\text { "Lumpy", thickly } \\
\text { bedded sites }\end{array}$ & $4 c^{8}$ & $\begin{array}{l}\text { Thick } \\
\text { lumpy } 2\end{array}$ & Inhomogeneous & Uniform & $>65$ & $50-100$ & $\begin{array}{l}\text { 4-6 orthogonally } \\
\text { oriented }\end{array}$ & none & $\begin{array}{l}\text { 1-3 for gravel- } \\
\text { sized matrix only }\end{array}$ & $5 a^{8}$ \\
\hline $\begin{array}{l}\text { "Lumpy", thinly } \\
\text { bedded sites }\end{array}$ & $4 b^{8}$ & $\begin{array}{c}\text { Thin } \\
\text { lumpy } 2\end{array}$ & Inhomogeneous & Uniform & $<65$ & $50-100$ & $\begin{array}{c}\text { 4-6 orthogonally } \\
\text { oriented }\end{array}$ & $\begin{array}{l}3-5 \text { for each unit } \\
\leq 30 \mathrm{~cm} \text { from } \\
\text { dating sample }\end{array}$ & $\begin{array}{l}\text { 1-3 for gravel- } \\
\text { sized matrix only }\end{array}$ & $5 b^{8}$ \\
\hline $\begin{array}{l}\text { "Lumpy", thickly } \\
\text { bedded sites }\end{array}$ & $4 d^{8}$ & $\begin{array}{l}\text { Thick } \\
\text { lumpy } 3\end{array}$ & Inhomogeneous & Non-uniform & $>65$ & $500-1000$ & $\begin{array}{c}4-6 \text { orthogonally } \\
\text { oriented }\end{array}$ & none & $\begin{array}{l}\text { 1-3 for each lump } \\
\text { mineralogy per } \\
\text { unit }\end{array}$ & $5 a^{8}$ \\
\hline $\begin{array}{l}\text { "Lumpy", thinly } \\
\text { bedded sites }\end{array}$ & $4 d^{8}$ & $\begin{array}{l}\text { Thin } \\
\text { lumpy } 3\end{array}$ & Inhomogeneous & Non-uniform & $<65$ & $500-1000$ & $\begin{array}{c}\text { 4-6 orthogonally } \\
\text { oriented }\end{array}$ & $\begin{array}{c}3-5 \text { for each unit } \\
\leq 30 \mathrm{~cm} \text { from } \\
\text { dating sample }\end{array}$ & $\begin{array}{c}1-3 \text { for each } \\
\text { lump mineralogy } \\
\text { per unit }\end{array}$ & $5 b^{8}$ \\
\hline
\end{tabular}

Table 2. Sampling for Associated Sediment.

${ }^{1}$ Sampling strategy and site character definition is governed by the most inhomogenous unit present. If one "lumpy 3" bed occurs within $35 \mathrm{~cm}$ of the sample, the whole sedimentary package is treated as a lumpy 3 site.

${ }^{2}$ Mineral compositions in the units within $35 \mathrm{~cm}$ of the dating sample:

Homogeneous = all a single mineral, e.g., all calcite or all quartz

Inhomogenous = mixed sediment with several mineral or rock fragment types, e.g., mixed limestone and bone, till with quartz sand and gravel-sized granite clasts

${ }^{3}$ Clast (grain) sizes in the units within $35 \mathrm{~cm}$ of the dating sample:

Uniform = all one or two $\beta$ size classes, e.g., all medium-coarse sand or all silt-fine sand

Non-uniform = several or a range of $\beta$ size classes, e.g., diamicton, breccia, most fossiliferous units, till

${ }^{4}$ Dosing units are sedimentary units within the $30 \mathrm{~cm} \gamma$ sphere of influence (Figures 4, 5):

10 (primary) dosing unit(s) = the one or two unit(s) touching the dating sample that contribute both $\beta$ and $\gamma$ dose to the external dose rate affecting the sample.

$2 o$ (secondary) dosing units = all units $\leq 35 \mathrm{~cm}$ from the dating sample that contribute only $\gamma$ dose to the external dose rate affecting the sample.

5 assuming that sediment matrix is sand-sized or smaller; larger matrix grain size requires larger sample mass.

${ }^{6}$ assuming that the clasts are collected separately from the matrix.

7 assuming that grains of only one mineral constitute all the components in the sedimentary unit(s).

${ }^{8}$ assuming that grains of several different minerals occur in the sedimentary unit(s) 


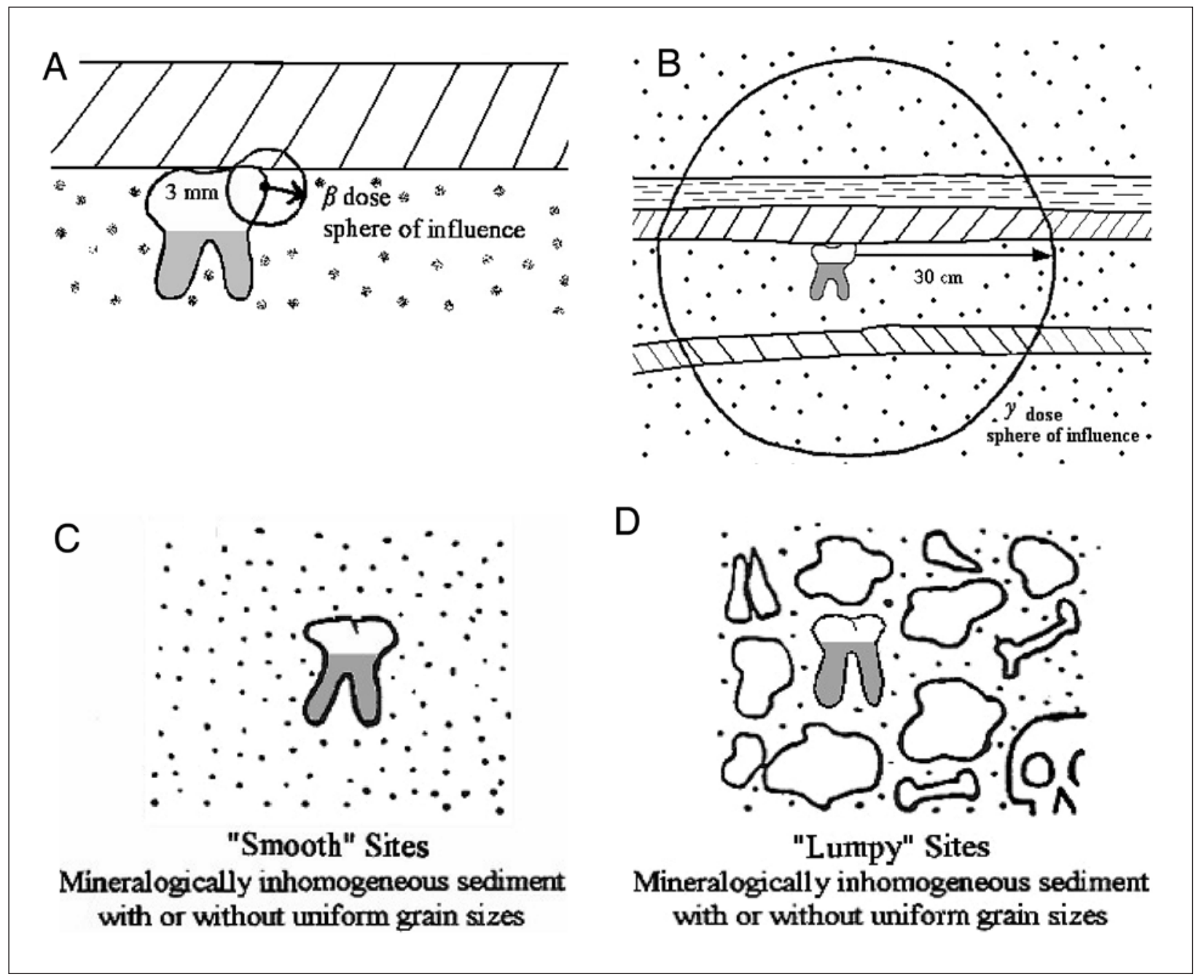

Fig. 4: Factors affecting the effective radiation dose field around dating samples.

Radiation can reach the dating sample from radioactive decay occurring within the sphere of influence for the particular radiation type: a. $\beta$ particles deliver to a sample a significant, but variable, component in the total radiation dose, both externally and internally. Since the penetration range for a $\beta$ particle averages 1-2 mm, comparable to the sample thicknesses, dose calculations must consider $\beta$ attenuation within the sample. The sphere of influence for the contributions from $\beta$ radiation will usually not include more than two or three sedimentary units.

b. Since $\gamma$ irradiation can penetrate $\sim 30 \mathrm{~cm}$, the sphere of influence for the contributions from $\gamma$ radiation can include several sedimentary units, which may produce very different dose rates.

c. In "smooth" sites with homogeneous sediment, the dose rate calculation is trivial.

d. In "lumpy" sites, different minerals or clasts within the sediment, which may contain different concentrations of radioactive elements, can contribute dose at very different rates.

In all situations, the $D_{\text {ext }}(t)$ calculation must volumetrically average the dose rate from each source relative to its importance and location within the sphere of influence each stratigraphic unit or sediment type. 
A.

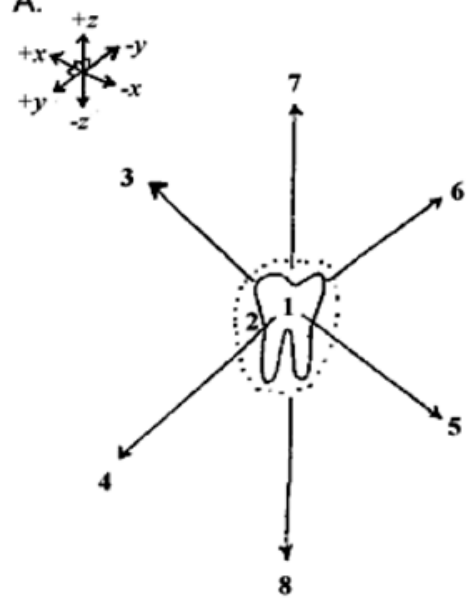

$\stackrel{\mathrm{cm}}{\mathrm{C}_{10}}$

\section{Dating sample}

2. Sediment immediately around the dating sample

Sediment samples $25-30 \mathrm{~cm}$ away from the dating sample relative to the local site grid

3. $+x$ direction, e.g., to the north 4. $+y$ direction, e.g., to the west 5. $-x$ direction, e.g., to the south 6. $-y$ direction, e.g., to the east 7. $+z$ direction, above 8. $-z$ direction, below
B.

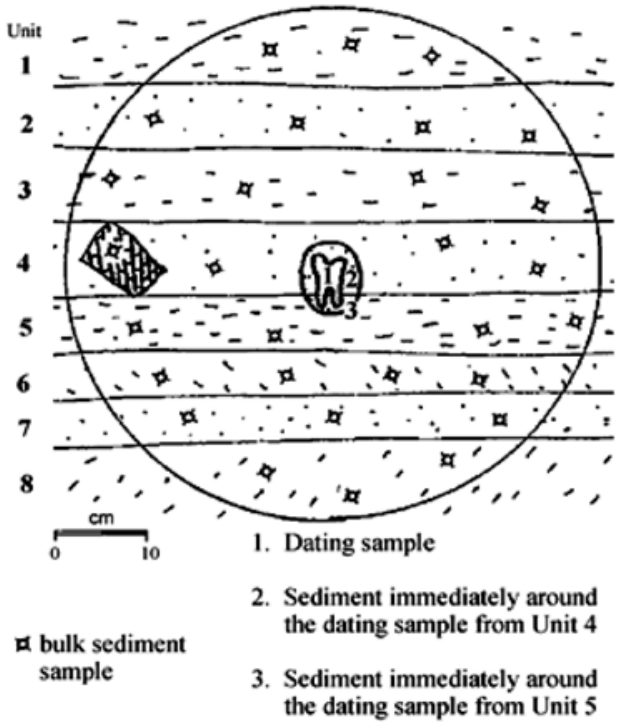

Fig. 5: Collecting protocols for associated sediment samples for ESR dating.

a. Thickly bedded homogeneous units ("Thick smooth" units, Table 2): Assuming that the dating sample lies at least $35 \mathrm{~cm}$ from the nearest sedimentary unit boundary, sediment should be collected from four to six of the six orthogonal positions. In pictured example, the associated sediments were collected from the six orthogonal positions that coincide with the site grid plan.

b. Thinly bedded or inhomogeneous units ("Thin smooth" units, Table 2): The sample for dating (1) sits within Units 4 and 5 (2, 3). In this circumstance, separate samples need to be collected from the two surrounding units. When sampling the surrounding sedimentary units, three to five sediment samples should be collected from each unit, distributing the samples throughout the unit as it falls within the $\gamma$ sphere of influence. Ideally for each unit, a few should come from along the cut face, one from behind, and one from in front of the cut face in order to sample a somewhat even distribution for each bed.

\section{ESR ANALYSIS}

Calculating an ESR age requires considering some 30 different parameters, which affect the accumulated dose, the internal and external dose rates. Although improved spectrometers and ancillary equipment have sped the process and improved precision somewhat, the basic ESR dating protocols were established in the 1980's. Standard analytical protocols for all mineralogies require powdered samples. Although some ESR labs have developed "nondestructive" analyses for tooth enamel (e.g., Robertson \& Grün, 2000; Miyake et al., 2000), even these cause some sample degradation.

\section{DETERMINING THE ACCUMULATED DOSE, $\mathrm{A}_{\Sigma}$}

For each sample, the accumulated dose, $A_{\Sigma}$, is determined using the additive dose method (Figure 6a). This requires about $0.2-0.5 \mathrm{~g}$ of pristine prepared mineral sample ( $\mathrm{Ta}-$ ble 1) in order to provide 10-15 aliquots of powdered, homogenized sample. Using fewer than 10 measurements causes significantly lower precision. Except for one, each aliquot is irradiated to a different precisely known artificial radiation dose, usually from a ${ }^{60} \mathrm{Co} \gamma$ source. The added doses used usually range from 0.1-10 Grays for the lowest added dose to 1-40 kGy for highest, depending on the sample's $A_{\Sigma}$. Older samples, those with higher $A_{\Sigma}$ 's, generally get higher doses. The selection of added doses does affect the curve fitting statistics, and hence, the precision for $A_{\Sigma}$ for enamel (e.g., Lee et al., 1997), and presumably for other materials as well. In the ramping technique, only 3-4 aliquots are used, but one or two are used to calibrate the spectrometer with each set of measurements, and two or three are successively irradiated to ever higher added doses (Blackwell, 2001).

After measuring the ESR signal heights for both the natural and irradiated aliquots, the added dose is plotted versus the signal intensity to produce a growth curve (Figure 6a). Usually, the points are weighted inversely with intensity (peak height). In some materials, however, signal subtraction is necessary to isolate the dating signal from the interference to measure an accurate peak 


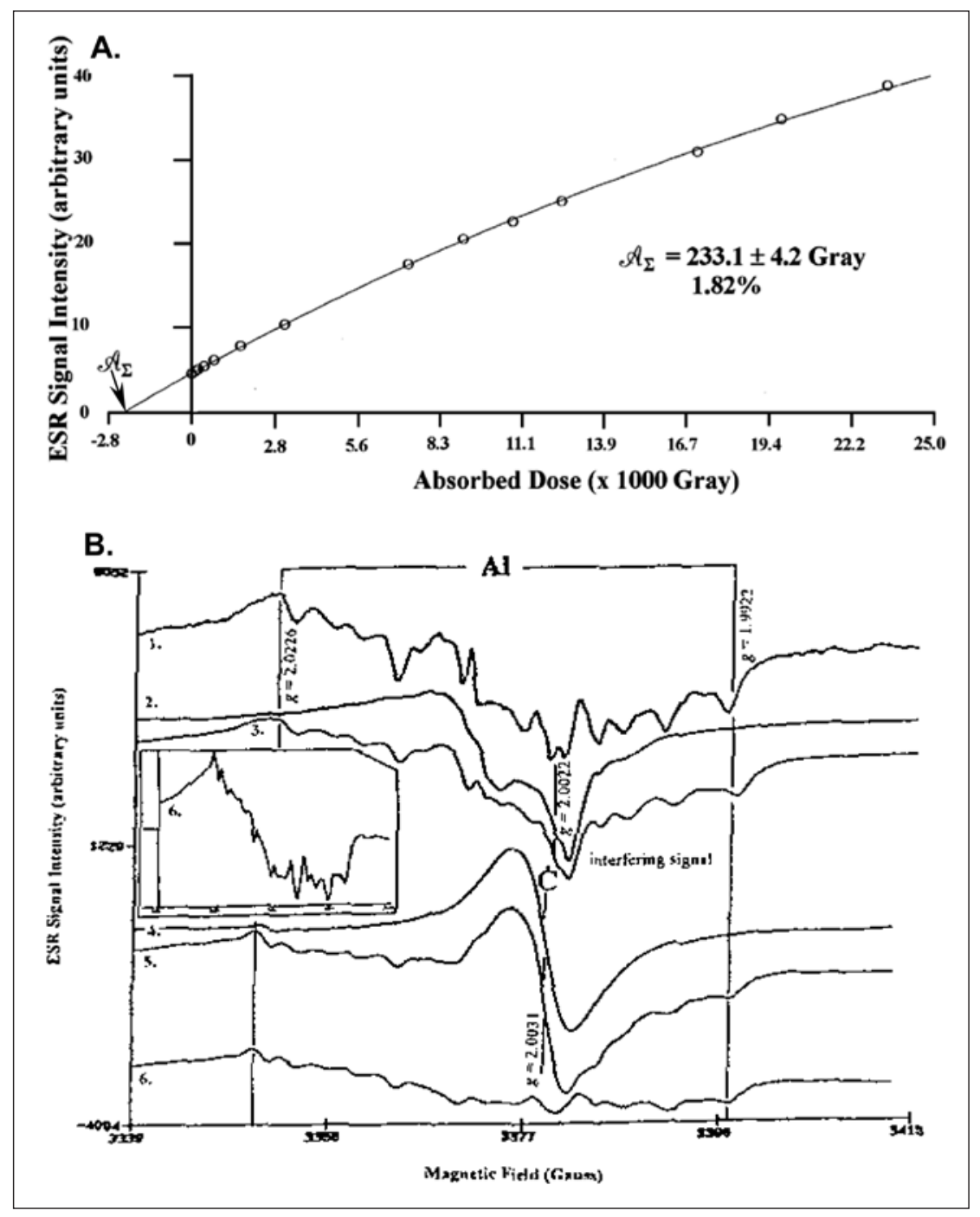

Fig. 6. Determining the accumulated dose, $A_{\Sigma}$.

The additive dose method is used to calculate the accumulated (or $\gamma$-equivalent) dose, $A_{\Sigma}$ :

a. Under artificial irradiation during analysis, the HAP signal saturates at its maximum intensity, $I_{\text {max }}$ Plotting the signal intensity versus the added radiation dose produces a growth curve. The $x$-intercept for this curve gives $A_{\Sigma}$. This bovid tooth from Treugolnaya Cave, Russia, has a substantial accumulated dose, as expected for a Middle Pleistocene site dating to OIS 11 (Blackwell et al., 2005a).

b. For signals suffering interference, signal subtraction is used to remove the interference:

Curve 1. A pure Al signal is unaffected by interference signals.

Curve 2. An organic radical signal, $\dot{C}$, interferes with the Al signal.

Curve 3. Unidentified interference signals affect the Al signal.

Curve 4. The Al and $\dot{C}$ signal in a natural archaeological sample.

Curve 5. The same sample as Curve 4 heated for 10 minutes at $320^{\circ} \mathrm{C}$ to zero the Al signal.

Curve 6. When Curve 5 is subtracted from Curve 4, the resulting signal shows the hyperfine splitting typical for the Al signal (see inset; modified from Blackwell, 2001).

height (Figure 6b). Despite controversies over measurement protocols, derivative spectra actually provide better resolution (Lyons \& Tan, 2000). Most evidence also suggests that deconvolution is not necessary for many dating peaks (e.g., Skinner et al., 2001a). The accumulated dose,
$\mathrm{A}_{\Sigma}$, required to produce the observed natural ESR signal intensity equals the $\mathrm{x}$-intercept for the growth curve. Within some materials, such as travertine, calcrete, and caliche, crystals may vary greatly in their $\mathrm{A}_{\Sigma}$. If some regions are at or near saturation, while others are younger, 
and hence not saturated, age underestimation may also occur, because the dose response is nonlinear near saturation (Figure $1 \mathrm{~b}$ ). This is not a problem for tooth enamel where linear behaviour persists to large doses (Brennan, 2000). Generally, $A_{\Sigma}$ can be measured with 0.8 to $5 \%$ precision depending on the spectrometer's calibration (Nagy, 2000), the radiation source calibration (Wieser et al., 2005), the sample's age and diagenetic state (e.g., Blackwell et al., 2005a).

\section{DETERMINING THE INTERNAL DOSE RATE, $\mathrm{D}_{\text {int }}(\mathrm{T})$}

To calculate the internal dose rate, $\mathrm{D}_{\text {int }}(\mathrm{t})$, the radiation sources (all U, Th, K, etc.) within the sample are measured (Figure 1d), usually using neutron activation analysis (NAA) or any geochemical technique able to measure elemental concentrations at the ppm-ppb range. Then, $\mathrm{D}_{\text {int }}(\mathrm{t})$ is derived from theoretical calculations. For samples containing $\mathrm{U}$ or Th, those calculations must also consider the increased radioactivity due to ingrowth of the $\mathrm{U}$ or Th daughter isotopes (Figure 7a) over time using an iterative procedure. Dint $(\mathrm{t})$ calculations also consider radiation attenuation by water within the sample, $\alpha$ and $\beta$ dose attenuation due to mineral density, and radon (Rn) loss for U- or Th-rich samples (Figure 7b; e.g., Brennan et al., 2000).

In samples, such as tooth enamel, bone, and fish scales, where the internal dose rate derives solely from $\mathrm{U}$ absorbed during its burial history, the calculated ESR age must account for $U$ uptake: Either the sample must be dated by $\mathrm{U}$-series or $\mathrm{U} / \mathrm{Pb}$ analysis, which allows a unique uptake model to be selected, or a U uptake model must be assumed. Without calibrating dates, four models are commonly used (Figures $7 c, 7 d$ ):

Early uptake (EU) assumes that the sample absorbed all its $U$ soon after burial, providing the youngest age given the accumulated dose, $A_{\Sigma}$, and external dose rate, $\mathrm{D}_{\text {ext }}(\mathrm{t})$.

Linear uptake (LU) assumes that the sample absorbs $U$ at a constant rate throughout its burial history, giving a median age.

Recent uptake (RU) assumes $U$ uptake very late in the sample's burial history, which reduces its internally generated dose, $\mathrm{A}_{\text {int }}$ to a minor contribution compared to $A_{\Sigma}$. This gives the maximum possible age.

Coupled uptake (CU) assumes that the enamel, dentine, cementum, and any attached bone in teeth absorb $U$ by different models. Often, it assumes LU for the enamel and EU for the dentine, cementum, and any attached bone, yielding ages somewhat younger than strict LU, but older than strict EU, models.

Other models have also been suggested (e.g., Ikeya et al., 1997). CU only applies to materials like teeth where two different phases absorb $U$ at different rates. In teeth, LU or CU ages often agree most closely with ages determined by other means for samples between than $80 \mathrm{ka}$ and $500 \mathrm{ka}$, but, within a site, the uptake model can vary, since it depends strongly on microenvironmental conditions (e.g., Blackwell et al., 2001b). "For fossils and other materials that uptake $U$ after deposition, TIMS or laserablation ${ }^{230} \mathrm{Th} /{ }^{234} \mathrm{U}$ analyses give coupled ESR- ${ }^{230} \mathrm{Th} /{ }^{234} \mathrm{U}$ calculations, which can constrain the U uptake history, as neither method can do independently (e.g., Eggins et al., 2003) For some older samples, it is still possible to use ${ }^{230} \mathrm{Th} /{ }^{234} \mathrm{U}$, providing the uptake has occured recently enough that the ${ }^{230} \mathrm{Th} /{ }^{234} \mathrm{U}$ ratios are not indistinguishable from secular equilibrium values. $\mathrm{U} / \mathrm{Pb}$ can date some uraniferous samples older than 1-2 Ma, but it has not yet been applied to delineate an ESR uptake model. U leaching or secondary $U$ uptake may also present problems for some samples, and hence, requiring complex models (Figure 7c; Blackwell et al., 2005b; Hoffman \& Mangini, 2003). Precisions for $\mathrm{D}_{\text {int }}(\mathrm{t})$ depend strongly on the precision for $\mathrm{U}$ concentration measurement. Delayed neutron counting (DNC) neutron activation analysis (NAA) can routinely provide precisions and detection limits as low as $\pm 0.02 \mathrm{ppm}$, whereas instrumental NAA averages $\pm 0.2 \mathrm{ppm}$ for precision and $\pm 1 \mathrm{ppm}$ for detection limits, which makes dating young samples impossible. Any other technique able to measure the $U$ at or below the ppb concentration level with better than \pm 0.02 ppm precision provides sufficient discrimination to yield reliable ESR ages.

\section{DETERMINING THE EXTERNAL DOSE RATE, $\mathrm{D}_{\text {EXT }}(\mathrm{T})$}

The external dose rate, $\mathrm{D}_{\text {ext }}(\mathrm{t})$, strongly affects the calculated ESR ages (Figure 8a), especially for samples with low internal dose rates, $\mathrm{D}_{\text {int }}(\mathrm{t})$, as is common for teeth from caves. Teeth from open-air sites tend to have larger internal dose rates, but the external dose rates, also can be more variable over the long term. Both types of sites need to be examined carefully to understand all the dynamic processes that affect the external dose rates.

To derive the total external dose rate, $\mathrm{D}_{\text {ext, } \Sigma}(\mathrm{t})$, four methods can be used:

1. TL dosimeters placed in the site to measure the current external dose rate, $\mathrm{D}_{\text {ext, } \gamma}\left(\mathrm{t}_{0}\right)$ from sedimentary $\gamma$ and cosmic sources over 0.5-2.0 years.

2. $\gamma$ spectrometers measure the current dose rate, Dext, $\gamma\left(\mathrm{t}_{0}\right)$ from sedimentary $\gamma$ and cosmic sources over 0.5-2 hours.

3. Bulk geochemical analysis, often by NAA, using powdered sediment collected in conjunction with the sample measures the $\mathrm{U}, \mathrm{Th}, \mathrm{K}$, and other significant radioisotope concentrations in any layers which may have 
contributed to the sample's $\mathrm{D}_{\text {ext, }}(\mathrm{t})$ (Figures 4,5$)$. The radioisotopic concentrations are used to mathematically calculate the current dose rates, $\mathrm{D}_{\text {ext, } \gamma}\left(\mathrm{t}_{0}\right)$ and $\mathrm{D}_{\text {ext, }, \beta}\left(\mathrm{t}_{0}\right)$ which include corrections for $\gamma$ and $\beta$ dose attenuation due to mineral density, and backscattering. Such $\mathrm{D}_{\text {ext, }}$ ${ }_{\Sigma}(\mathrm{t})$ calculations also require a measurement for, or assumptions about, $\mathrm{D}_{\text {cos }}(\mathrm{t})$, the cosmic dose rate (Figures $8 \mathrm{~b}, 8 \mathrm{c}$ ) for samples buried less than $10 \mathrm{~m}$ and also the average sedimentary water concentration to correct for radiation attenuation by sedimentary water (Figure $8 \mathrm{~d}$ ). In sites with thinly layered deposits or inhomogeneous sediment, $\mathrm{D}_{\text {ext, } \Sigma}(\mathrm{t})$ calculations ideally should consider each unit or sediment component individually by determining volumetrically averaged dose contributions (Figure 4b).

4. An isochron age for a large sample may obviate the need for a $\mathrm{D}_{\text {ext, },}(\mathrm{t})$ calculation, because it gives both the sample age, $\mathrm{t} 1$, and $\overline{\mathrm{D}}_{\text {ext, } \Sigma}(\mathrm{t})$ the time-averaged external dose rate, simultaneously (Figure 9).

For adjacent U-rich or Th-rich layers or sediment components, the measurement or calculation is corrected for possible $U$ uptake, $U$ daughter isotope ingrowth, and potential Rn loss (e.g. Figure 7; Blackwell \& Blickstein, 2000).

Assuming that $\mathrm{D}_{\text {ext }}(\mathrm{t})$ has remained constant throughout the burial history, as many early studies did, can be naive. Changing water or radioactive element concentrations in the sediment (Figure 8d; e.g., Olley et al., 1997), increasing burial depth (Figure 8b), or variable $\mathrm{D}_{\cos }(\mathrm{t})$, among others, can all affect the $\mathrm{D}_{\text {ext, } \Sigma}(\mathrm{t})$ experienced by the sample, requiring that $\mathrm{D}_{\text {ext }, \beta}\left(\mathrm{t}_{0}\right)$ and particularly $\mathrm{D}_{\text {ext, },}\left(\mathrm{t}_{0}\right)$ be corrected for any such significant variations. At sites where sedimentary water concentration variations can be significant, or where sediment accumulation or deflation can alter the depth of sediment cover, these considerations become significant, but not insurmountable.

In using geochemical analysis (e.g., NAA) at sites with very inhomogeneous sediment units ("lumpy" sites), the inhomogeneity in the dose field (Guibert et al., 1998) requires volumetric analysis in which the contribution from each component (Figure 1d, 8d) depends on its abundance in order to calculate the actual contribution to $\mathrm{De}_{\mathrm{xt}, \Sigma}(\mathrm{t})$ from different components or layers within the $\beta$ and $\gamma$ "spheres of influence". That still, however, does not consider the potential changes in $\mathrm{D}_{\text {ext, } \Sigma}(\mathrm{t})$ due changes in radioisotopic concentrations within the sedimentary components. In lumpy sites, sedimentary components which may be able to absorb $U$ (e.g., peat, teeth, bones, mollusc shells) can constitute a significant sedimentary fraction. If they can absorb $\mathrm{U}, \mathrm{D}_{\text {ext, }}(\mathrm{t})$ will probably have changed with time, because,
1. Components such as teeth and bone only absorb $\mathrm{U}$, not all its daughters which ingrow later (Figure 7a).

2. If the uptake occurred early in the sediment's history, its effect will be greater than if it occurred recently. This requires that $U$ uptake into the sediment be modelled analogously to that into teeth (see $\mathrm{D}_{\text {int }}(\mathrm{t})$ models above; Figure 7c).

3. $\mathrm{U}$ or other soluble daughters may have been leached, or Rn may have diffused (Figure 7b), from these components, requiring modelling to assess the effect on $\mathrm{D}_{\text {ext, },}(\mathrm{t})$ (e.g., Pike \& Hedges, 2001; Figure 7c).

4. More than one discrete uptake or loss event may have affected these components (Figure 7c).

These sedimentary processes can produce significant differences in the calculated $\mathrm{D}_{\text {ext, } \Sigma}(\mathrm{t})$ and ages. Therefore, the isochron method is preferred whenever possible, because the sample acts as its own dosimeter, theoretically compensating for inaccuracies due any change in $\mathrm{D}_{\text {ext, } \Sigma}(\mathrm{t})$.

Precision in ESR dating depends on the method used to measure $\mathrm{D}_{\text {ext, } \Sigma}(\mathrm{t})$ and the relative radioactive element concentrations. For $\gamma$ and TL dosimetry, precision tends to average $3-10 \%$, whereas for sedimentary analysis, uncertainties normally range from 5 to $15 \%$. Precision for $\overline{\mathrm{D}}_{\text {ext. } \Sigma}(\mathrm{t})$ in isochron analysis will exceed that associated with the isochron age, because $\overline{\mathrm{D}}_{\text {ext, } \Sigma}(\mathrm{t})$ is derived from the age, rather than vice versa. The different measurement protocols do often yield somewhat different estimates for $\mathrm{D}_{\text {ext, },}(\mathrm{t})$ (Blackwell et al., 2000).

\section{THE ISOCHRON METHOD}

Isochrons have been applied mainly to teeth (Figure 9), but also fault gouge minerals and stalagmites. With the isochron method, a sample that can yield at least five subsamples is analyzed by standard ESR analysis. If the accumulated doses, $A_{\Sigma, i}$ plotted against the time averaged internal dose rate, $\overline{\mathrm{D}}_{\text {int, } \mathrm{i}}(\mathrm{t})$ for each subsample, $i$, give a straight line, its slope equals the sample's age, $t_{1}$, while the $y$-intercept yields the accumulated dose due to external sources, Aext, from which can be derived the time-averaged external dose rate, $\overline{\mathrm{D}}_{\text {ext, } \Sigma}(\mathrm{t})$ (Figure 9a).

In teeth, the method gives a family of lines which converge on Aext, but whose ages and $\overline{\mathrm{D}}_{\text {ext, },}(\mathrm{t})$ each depend upon the $U$ uptake model used to calculate $\bar{D}_{\text {int, }}(t)$ (Figures $9 \mathrm{~b}, 9 \mathrm{c})$. Tests have shown that, if the isochron has a high $\mathrm{R}^{2}$ for the regression, the slope gives an age consistent with other dating methods (Blackwell et al., 2002a). The isochron method is limited to samples whose internal dose rate, $D_{\text {int }}(t)$, constitutes a significant fraction of $D_{\Sigma}(t)$, effectively requiring the sample to contain $\geq 2 \mathrm{ppm}$ $\mathrm{U}$. If samples have lost $\mathrm{U}$ or gained $\mathrm{U}$ in more than one event, however, isochron analyses may give erroneous ages and/or $\overline{\mathrm{D}}_{\text {ext, } \Sigma}(\mathrm{t})$ values (Figure 9d; Blackwell et al., 2001a). Precisions for isochron ages and $A_{\text {ext }}$ can range 
A.

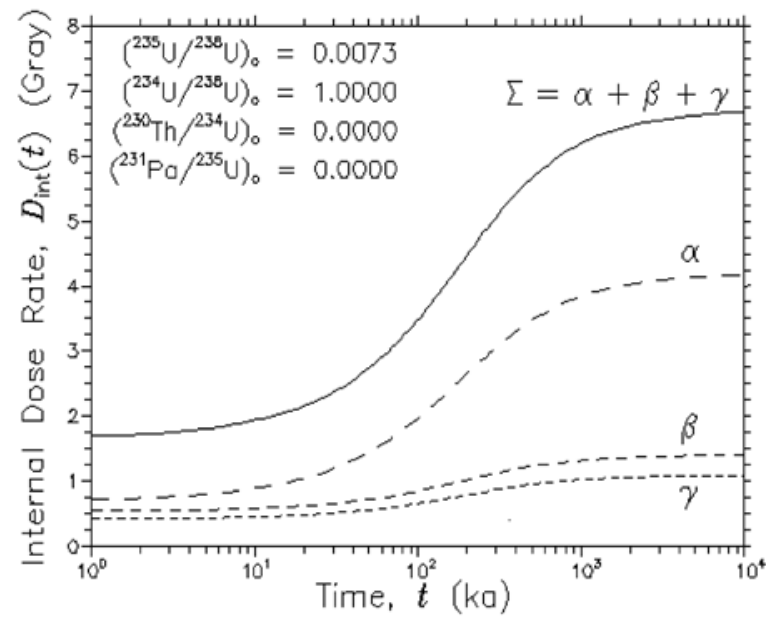

C.

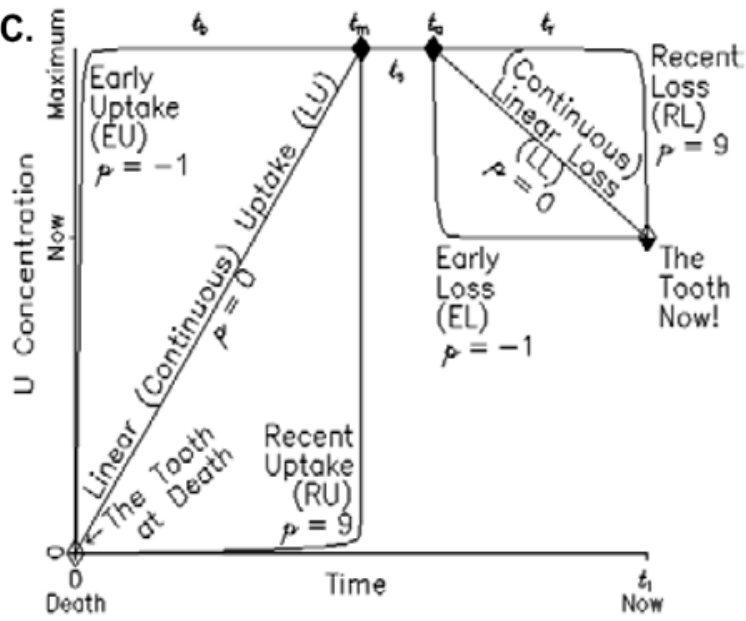

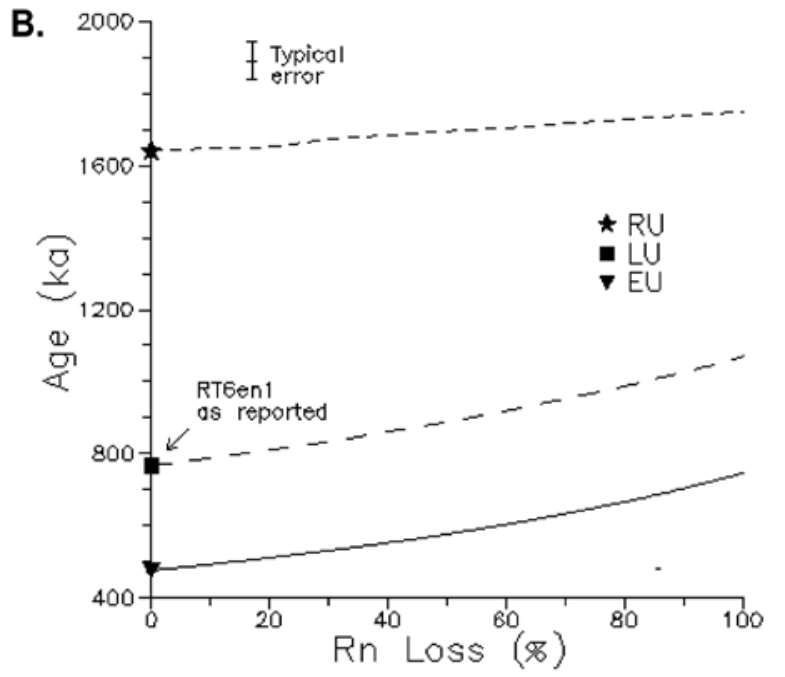

D.

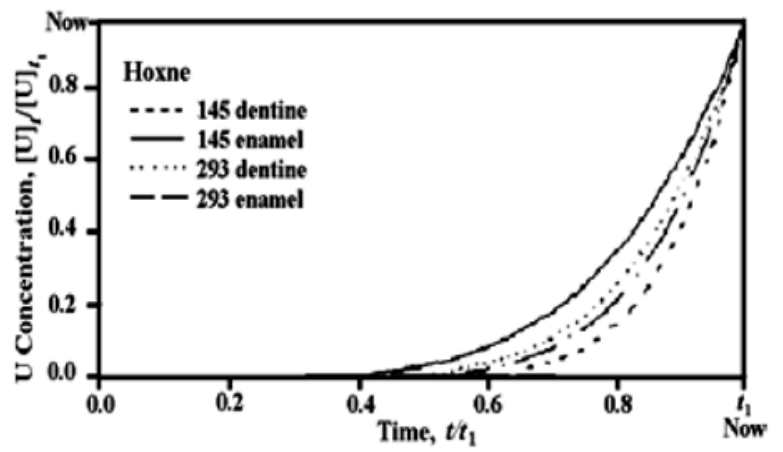

Fig. 7: Factors affecting the internal dose rate, $D_{\text {int }}(t)$.

For bones, teeth, molluscs, and other materials containing or capable of absorbing $U$, $U$ uptake must be measured or modelled. For minerals or fossils capable of losing $U$ or other $U$ daughter products by leaching or degassing, these must also be modelled or measured:

a. $D_{\text {int }}(t)$ increases as the sample ages simply from ingrowth of the $U$ daughter isotopes. This plot assumed an early uptake model $U$ absorption of 10 ppm, with no initial Th or Pa.

b. Radon (Rn) gas, produced when $U$ decays, can escape from samples during diagenesis and fossilization, causing $D_{\text {int }}(t)$ to decrease, and therefore, affecting the accuracy in the calculated ages. Assuming $0 \% \mathrm{Rn}$ loss will not contribute significant errors to age calculation for most samples, except those with very high U concentrations. In this mammoth molar from a pond deposit in Hungary, the uptake model significantly affects the age calculation, because the dentine contains relatively high $U$ concentrations, producing significant differences in the various calculated model ages.

c. A combined model for U uptake and leaching: The fossil absorbs all its U immediately after death in the early uptake (EU) model, but it absorbs almost no $U$ until just before attaining its maximum $U$ concentration in the recent uptake (RU) model. Under linear uptake, the fossil absorbs $U$ continuously and constantly throughout the uptake time, and linear leaching assumes an analogous continuous, constant $U$ loss through the leaching period. Under early leaching (EL), the fossil loses $U$ in a geological instant some time before the fossil is discovered, whereas under recent leaching, the loss occurs just before discovery.

d. U uptake in teeth from Hoxne, England: Recent uptake models are applicable in some situations.

More complex models can be devised by combining several uptake and leaching events (adapted from Blackwell, 2001). 

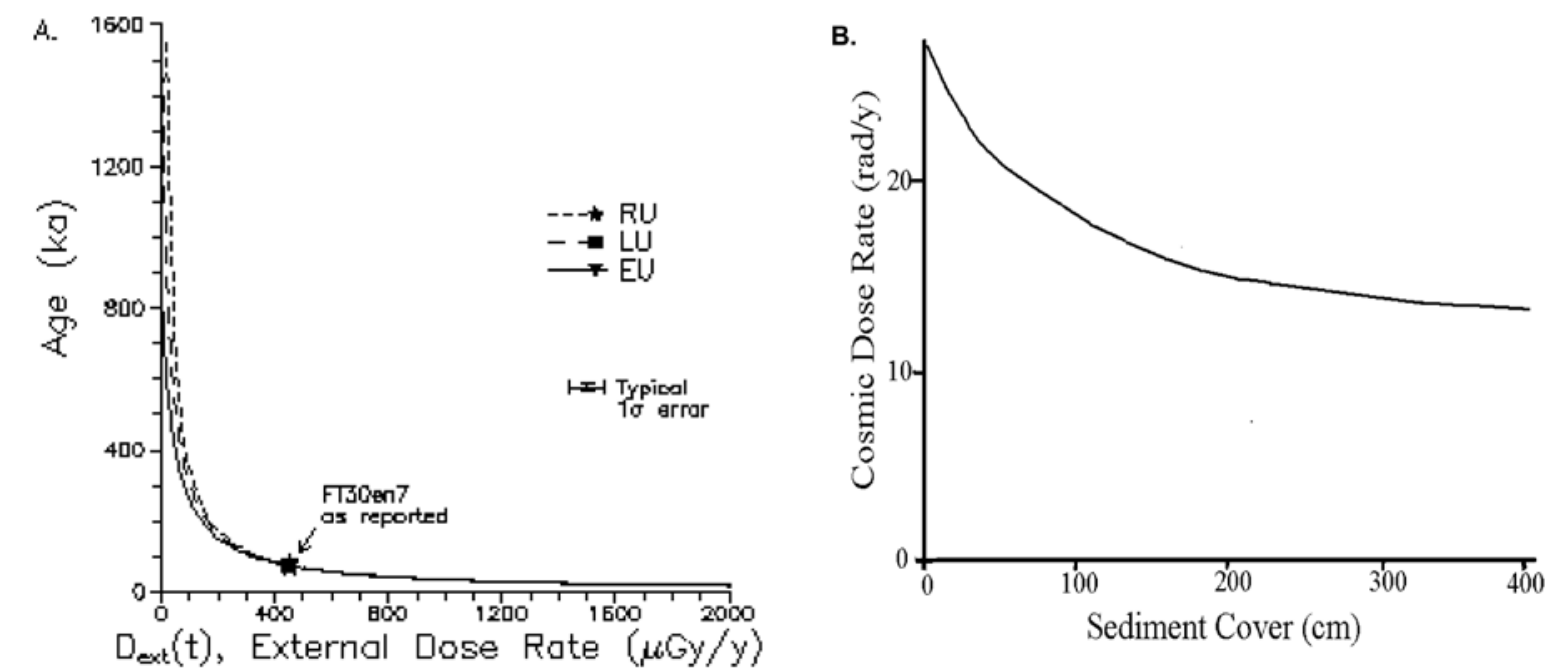

c.
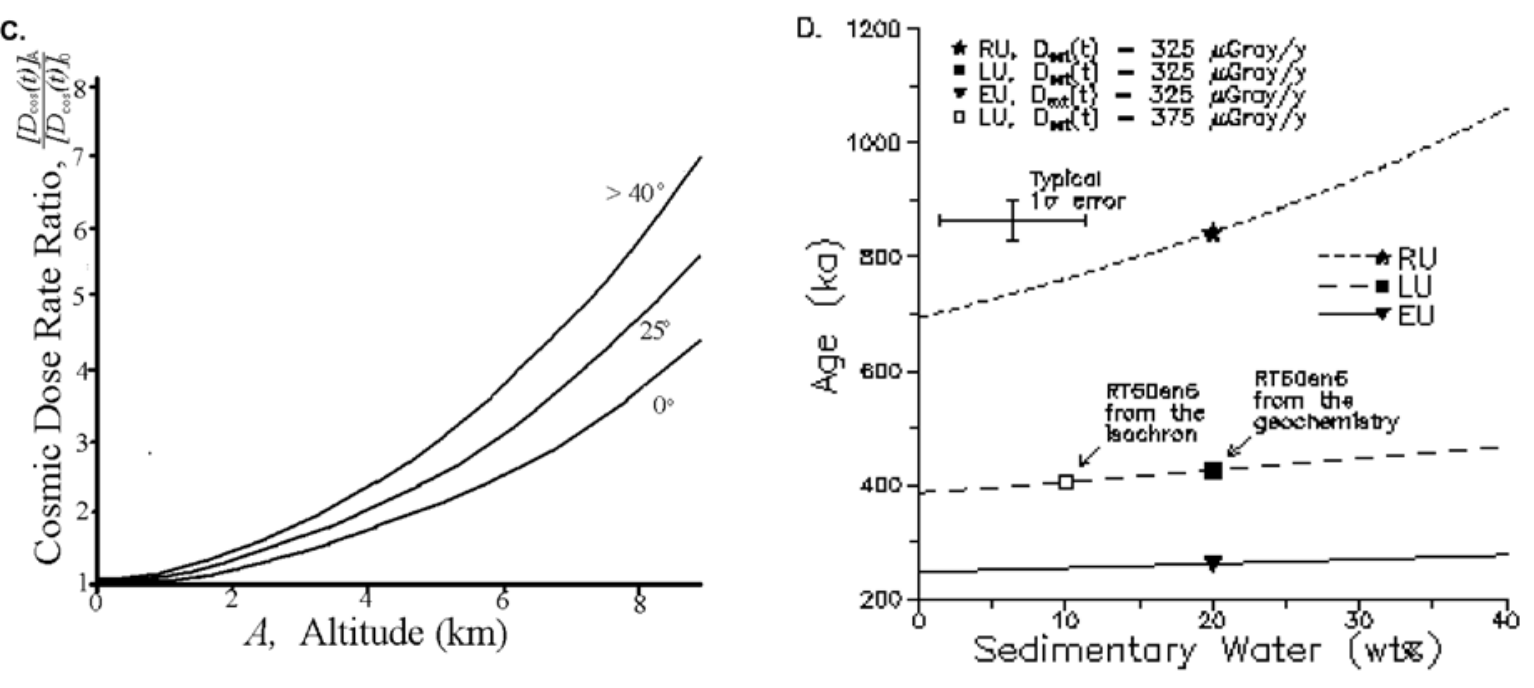

Fig. 8: The effect on ESR ages from the external dose rate, $D_{\text {ext }}(t)$.

$\operatorname{Dext}(t)$ is a function of many variables, including the water in the sediment and the cosmic dose impacting the sample:

a. Miscalculated $D_{\text {ext }}(t)$ 's can dramatically affect the calculated ages, especially for the RU ages. As the external dose rate increases, all the model ages decrease exponentially approaching $18 \mathrm{ka}$ at $2.0 \mathrm{mG} / \mathrm{y}$. A $200 \mu \mathrm{G} y / y(40 \%)$ decrease in the measured $D_{\text {ext }}(t)$ would reduce the calculated ages by approximately 13-15 ky ( 25\%), whereas a $200 \mu \mathrm{Gy} / \mathrm{y}$ (40\%) decrease would introduce a 26-32 ky ( $50 \%)$ increase in the calculated ages. These are insignificant compared to the $2 \sigma$ uncertainties in the age calculation (Blackwell et al., unpublished data).

b. As sediment depth increases above a sample, the cosmic dose contributes less to the total external dose rate. For samples covered by $10 \mathrm{~m}$ of sediment, the cosmic dose is negligible.

c. At higher altitudes and higher latitudes, the cosmic dose increases.

d. Sedimentary water attenuates the external dose reaching the tooth. As the sedimentary water concentration increases, the external dose rate, $D_{e x t}(t)$, decreases, but the calculated ESR age increases under all uptake models. Generally, changing the sedimentary water concentration by $\pm 5-10 w t \%$ does not significantly affect the calculated ages, especially for samples where $D_{\text {ext }}(t)$ represents a small percentage of the total dose rate, $D_{\Sigma}(t)$, as here. If, however, the sedimentary water concentration changes by $> \pm 10 w t \%$, the model ages will exceed the reported values by more than the $2 \sigma$ uncertainty in many samples, especially under the RU model, as seen here. Using the water concentration suggested by the $D_{\text {ext }}(t)$ from the isochron analysis does not produce a significant change (after Blackwell et al., 2005a). 


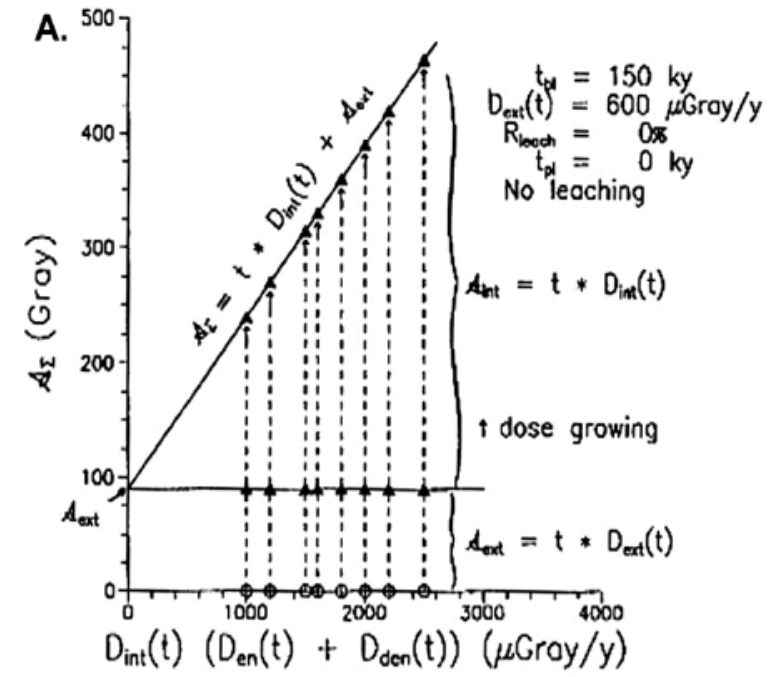

B. T87, Layer $\mathrm{H} 2$,

$$
\text { Bau de l'Aubesier }
$$

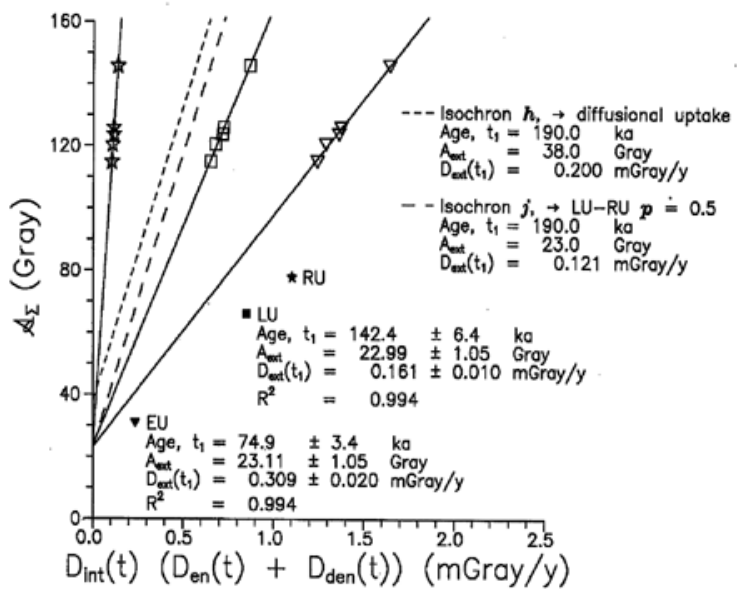

c. T70,

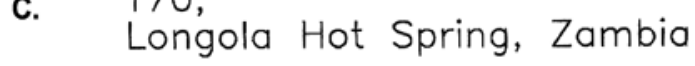

D. QT11, Layer H2,
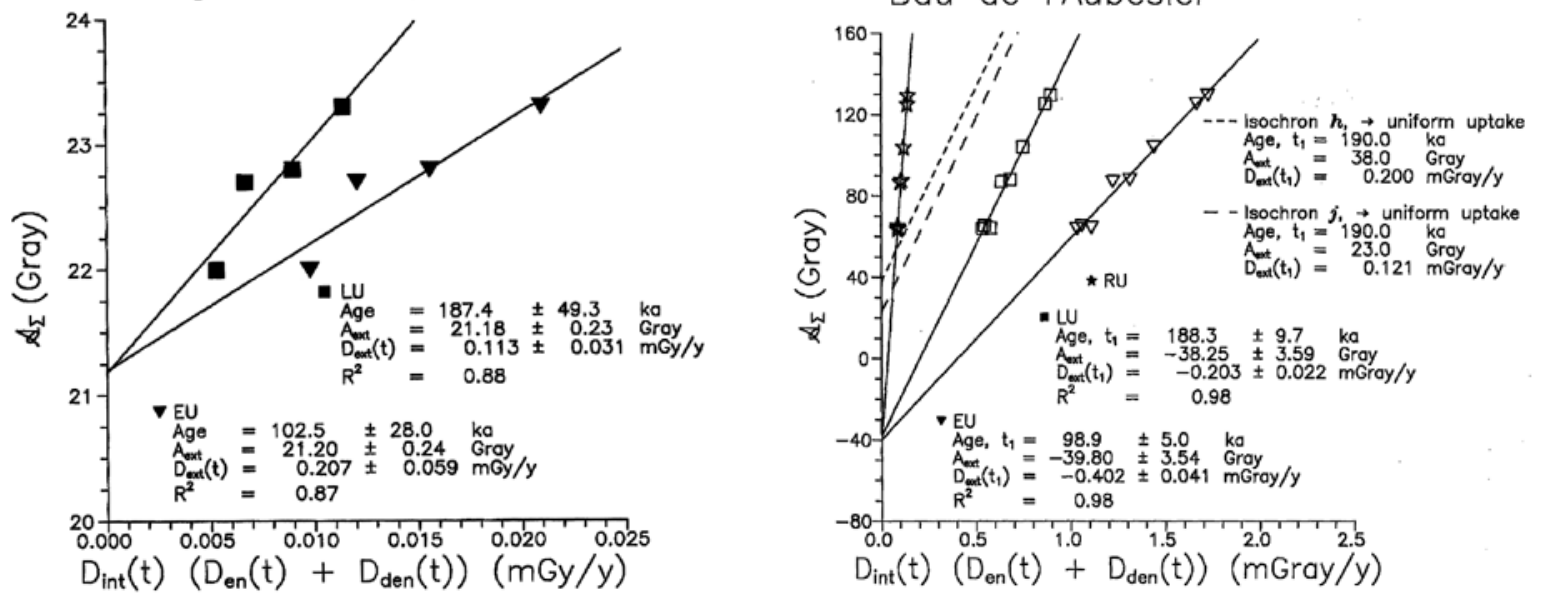

Fig. 9. ESR isochrons.

a. A theoretical plot: When the total accumulated dose, $A_{\Sigma, i}$, for each subsample, $i$, is plotted versus the time-averaged internal dose rate, $\bar{D}_{i n t, i}(t)$, the slope of the line gives the sample's age, $t$, while the $y$-intercept represents the external accumulated dose, $A_{\text {ext }}$

b. A plot for a tooth from Bau de l'Aubesier, Provence: In practice, each uranium uptake model produces a line, which all converge on $A_{\text {ext }}$. Isochron analysis can yield ages with uncertainties as low as $4 \%$.

c. An isochron for a tooth from tufa deposit associated with a thermal spring and lake at Longola, Zambia.

d. If a sample, such as this tooth, has experienced U leaching or a second uptake event, the isochron's intercept often becomes negative. In this example, the secondary uptake event must have occurred recently, because the isochron age agrees well with ${ }^{230} \mathrm{Th} / 234 \mathrm{U}$ age on adjacent stalagmitic horizons.

$\bar{D}_{i n t, i}(t)$, and $A_{\text {ext }}$ all depend on the U uptake model selected after the first iteration of this technique (adapted from Blackwell et al., 2002a). 
as low as $3-4 \%$, but normally tend to be less precise than standard ESR analyses, while minimum uncertainties for $\overline{\mathrm{D}}_{\text {ext, } \Sigma}(\mathrm{t})$ tend to be $\sim 5-6 \%$.

Because the isochron method averages $\mathrm{D}_{\text {ext, } \Sigma}(\mathrm{t})$ over the entire burial history, isochron analysis automatically corrects for any changes in $\mathrm{D}_{\text {ext, } \Sigma}(\mathrm{t})$ which may have occurred. By greatly reducing the need to measure $\mathrm{D}_{\text {ext, }}(\mathrm{t})$ in situ or to assume that it has remained constant, it can date samples from environments where $\mathrm{D}_{\text {ext, } \Sigma}(\mathrm{t})$ are likely to have changed in response to complex sedimentological changes, such as open-air environments. Isochrons can also date samples from sites that have been destroyed or are otherwise inaccessible, especially samples in museum collections.

If an independent method (e.g., TL or $\gamma$ dosimetry) can be used to measure $\mathrm{D}_{\text {ext, } \Sigma}(\mathrm{t})$, and if $\mathrm{D}_{\text {ext, } \Sigma}(\mathrm{t})$ can be shown to have been constant throughout time at the site by geological studies or an independent date, the isochron method can instead determine the $\mathrm{U}$ uptake history. Since the isochron calculation gives $\mathrm{A}_{\text {exte }}$, which must equal the product of the age, $t_{1}$, with $\mathrm{De}_{\mathrm{xt}, \Sigma}(\mathrm{t})$ the isochron's slope that matches this age represents the "correct" isochron and uptake model for the sample.

\section{ESR MICROSCOPY AND OTHER NEW TECHNIQUES}

In ESR microscopy, an ESR spectrometer has been modified to scan across a solid mineral surface to measure the spin concentrations for a preset signal. With specialized analytical programs, 2D, 3D, and 4D ESR imaging is now possible, some of which are combined with other systems such as electrically stimulated luminescence (ESL), NMR and CT (e.g., Miki et al., 1996; Mizuta et al., 2002). ESR microscopy is still being explored to understand its full potential, but it shows great promise in studying fossil diagenesis, mapping crystal growth and defects, among other applications. Currently, it works best for materials with very strong ESR signals, such as tooth enamel (e.g., Oka et al., 1997), bone (Schauer et al., 1996), coral, gypsum, mollusc shells, aragonite, and barite.

Portable ESR dosimeters and spectrometers are used to assess nuclear radiation accidents on site (e.g., Oka et al., 1996). Geoscientists can also use them in the field. In the field, such technology would help to recognize reworked fossils, to aid in selecting the best samples for dating, and to assess the effect of site inhomogeneity on the samples. Eventually, such technology may even allow preliminary age estimates while still in the field.

\section{APPLICATIONS AND DATABLE MATERIALS IN KARST SETTINGS}

Within karst settings, ESR can date materials that might provide valuable insight into a cave's or a karst system's history. Dating teeth, molluscs, ratite egg shells, authigenic carbonates or salts can delineate depositional histories and rates. Dates on authigenic cements may date diagenetic events or hydrological changes. Dating fossils, such as molluscs, teeth, and molluscs dates changes in biological diversity and groundwater chemistry. Dating burnt flints or hearth sands from archaeological sites or fossils from karst deposits can indicate the age for associated geomorphic surfaces and hint at paleoclimatic histories. Typical karst process, however, can cause all fossils, especially loose teeth, ratite egg shells, and molluscs, to be reworked (Figure 10).

\section{MOLLUSCS, RATITE EGGS SHELLS, OSTRACODES, AND OTHER CARBONATE FOSSILS}

In caves, open-air spring deposits, and karst fissure fills, dating mollusc shells found in the sediment (Table 2) can provide diverse information for Quaternary karst studies. Mollusc shells, however, act as open systems for U, although the moderate discordance between measured ${ }^{230} \mathrm{Th} / 2^{34} \mathrm{U}$ and ${ }^{231} \mathrm{~Pa} /{ }^{235} \mathrm{U}$ ratios suggests that most $\mathrm{U}$ uptake accompanies sedimentation.
Aragonitic mollusc shells normally show five ESR peaks (Figure 11), but calcitic molluscs have more complex spectra. For the calcitic peaks at $g=2.0018,2.0007$, and 1.9976, trap density is related to $\mathrm{Mg} / \mathrm{Ca}$ ratios, which can change with diagenesis, secondary mineralization, and fossilization, making them unsuitable for dating some species. Generally, either the peaks at $\mathrm{g}=2.0012$ and 2.0007 in calcitic shells and the peak at $\mathrm{g}=2.0007$ in aragonitic shells are the most reliable, but that must be tested for each species individually, because complex peaks do occur and peaks other than that at $g=2.0007$ may be light sensitive (Bartoll et al., 2000). Secondary mineralization can cause interference that affects $A_{\Sigma}$ measurement and age calculation. Signal lifetimes vary significantly depending on the peak and species (e.g., Blackwell, 1995, Table 2). Some species show inflection points in their growth curves, making it difficult to select an appropriate set of added doses for measuring $\mathrm{A}_{\Sigma}$ (e.g., Shih et al., 2002). Schellmann and Radtke (2001) advocated using a plateau technique with 40-60 irradiation steps to maximize accuracy in the growth curves.

Petrographic or geochemical analysis should accompany any ESR date to avoid remineralized and recrystallized samples. Contamination from $\mathrm{Mn}$ peaks often requires 


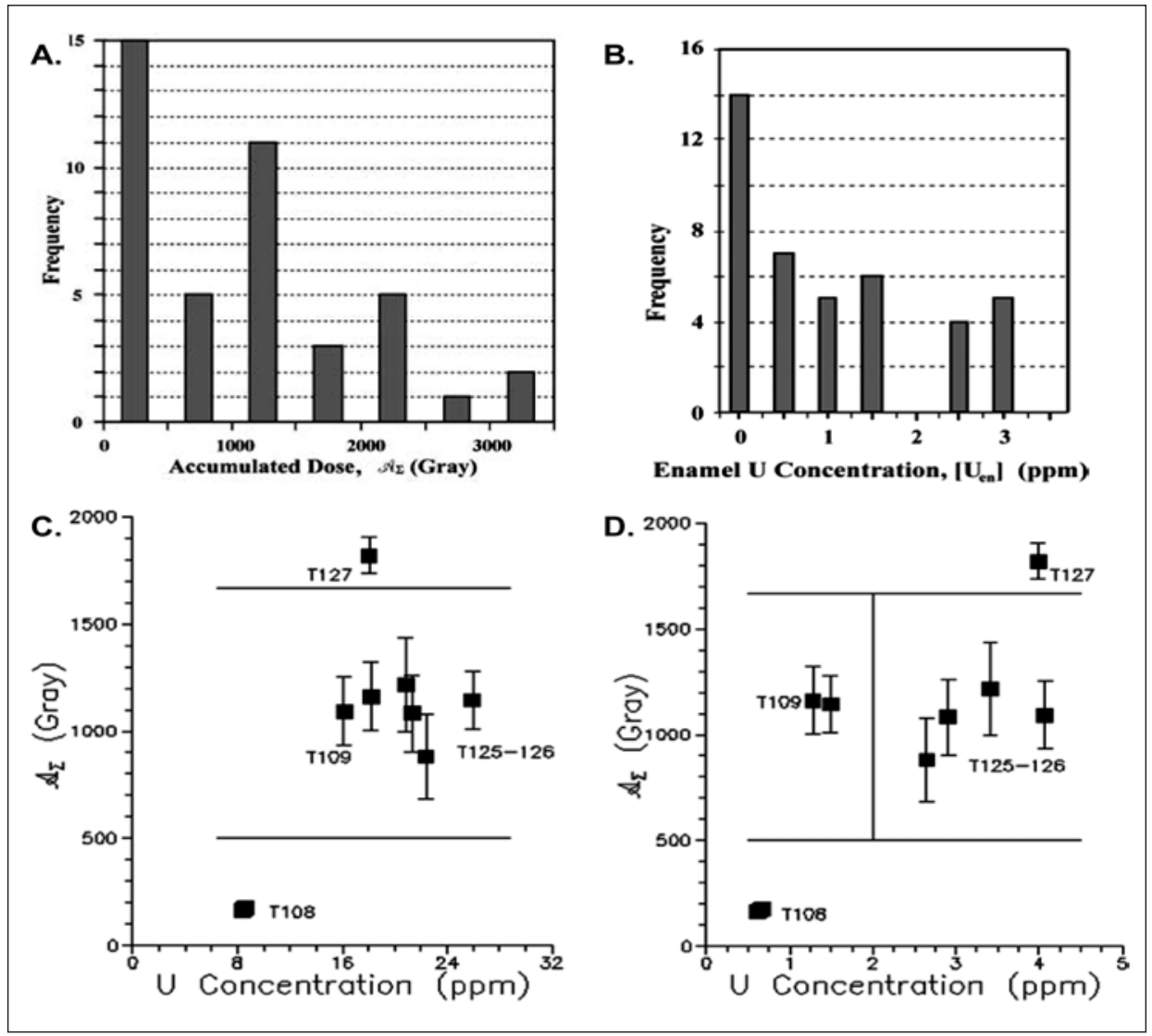

Fig. 10: Tests to check for reworked fossils.

For teeth from Swartkrans, South Africa:

a. The accumulated dose $\left(A_{\Sigma}\right)$ histogram clearly reveals at least three different populations of teeth.

$b$. The enamel $U$ concentration histogram shows at least two populations.

c. Plotting $A_{\Sigma} v$ s. enamel $U$ concentration reveals four distinct populations.

d. Plotting $A_{\Sigma} v$ s. dentinal $U$ concentration shows three different populations well separated from each other. Such plots delineate populations of teeth that have experienced different environmental conditions, one indication for reworking among samples from the same units (after Blackwell, 1994).

overmodulation to discriminate the dating peaks. Due to $\mathrm{U}$ uptake, modelling is required for samples that cannot be analyzed by coupled ESR- ${ }^{230} \mathrm{Th} / 2^{34} \mathrm{U}$ dating. In some fresh and hypersaline systems, the $\left({ }^{234} \mathrm{U} /{ }^{238} \mathrm{U}\right)$ o ratio may also need to be measured or modelled. For each species and signal, the $\beta$ efficiency factor, $\kappa_{\beta}$, must be measured. Long-term signal fading may also need to be considered, depending on the peak and its thermal stability.

Specimens found in life position give the most reliable results, although that does not guarantee that reworking has not occurred. Larger species are preferred so that each subsample represents a single individual (Table 1), but several shells can be combined from a smaller species, assuming that none have been reworked. Fragmentary samples still need to be speciated. Since species effects do occur, submitting two or three different species from each unit can increase dating precision and accuracy. Good agreement between ESR, TL, ${ }^{14} \mathrm{C}$, and AAR (amino acid racemization) ages has occurred in studies with Hendersonia and Allogona using $g=2.0007$, in Lymnaea baltica and Cerastoderma glaucum using $g=2.0012$. Thermal stabilities in Monauha caucaicala significantly exceeded those in marine molluscs. For untested species, $\sim 100 \mathrm{~g}$ of pristine shell are needed to perform the necessary signal stability and calibration tests (Balckwell, 2001).

Applications in karst systems have been rare, but terrestrial and freshwater molluscs do give reasonable ESR ages. For example, Molod'kov (2001) reported ages of $393 \pm 27 \mathrm{ka}$ for Layer $5 \mathrm{~b}$, and $583 \pm 25 \mathrm{ka}$ for Layer 7a for terrestrial molluscs preserved in the Lower Paleolithic site at Treugol'naya Cave, Russia. 
In ratite egg shells, two signals with good sensitivity exist. Although attempts have been made to date extinct birds, recent stability tests showed a very short signal lifetime, which would severely limit their application for sites older than $30 \mathrm{ka}$ (Skinner et al., unpublished data).

\section{AUTHIGENIC CARBONATES, SPELEOTHEM TRAVERTINE, CALCRETE, CALICHE}

Speleothem and travertine from springs, as well as in swamps and shallow hypersaline lakes, contains calcite or aragonite with several strong signals. Unfortunately, many travertines and some speleothems also contain high organic concentrations that can add interference peaks. Nonetheless, ESR dating of speleothem, travertine, and other authigenic carbonates allow detailed paleoenvironmental determinations, and may document prehistoric human activities.

How post-sedimentary processes affect the ESR signals in authigenic carbonates (Blackwell, 1995, Table 2) is still not well understood. Although most travertine spectra (Figure 12) resemble those for speleothems, which have been extensively studied, other peaks do occur. The humic acid signal at $\mathrm{g}=2.0040$ does not appear accurate for dating. In Mn-rich samples, the peak at $\mathrm{g}=2.0022$ yielded reliable ages, but needs testing for annealing behaviour and replicability before general application. The most reliably measured peak occurs at $g=2.0007$, while peaks other than that at $\mathrm{g}=2.0036$ may show light sensitivity (Bartoll et al., 2000). Although many authigenic carbonates lack the peak at $g=2.0007$, carefully sampling densely crystallized calcite can increase the success rate. Reliable ages have been found for some travertines, when compared against ${ }^{14} \mathrm{C}$ or $\mathrm{U}$ series ages. For some pisolites, calcrete, and caliche, contamination causes complex interference signals that affect accuracy, but preannealing samples before analysis may improve the results (Skinner, 2000).

Because most authigenic carbonates can experience remineralization, secondary mineralization or cementation, petrographic, SEM, XRD, or similar analyses should complement the ESR dating analysis to ensure viable geological conclusions. Otherwise, sample preparation is fast, requiring only powdering and a dilute acid leach to remove any transitory peaks induced by the grinding.

Relatively few ESR studies (e.g., Whitehead et al., 2002) have systematically examined travertine or other authigenic carbonates after problems with the applications were found in the 1990's. Attempts to date the spring travertines from Vertésszőlös, Hungary, failed to reveal a datable signal without interference (Skinner et al., unpublished data). Modern signal subtraction and multiband studies might resolve some problems and improve the reliability for these applications (Kinoshita et al., 2004).

\section{HYDROXYAPATITE (HAP), VERTEBRATE FOSSILS} AND CRUSTACEAN CHITIN

ESR analysis can date hydroxyapatite (HAP), because a single radiation-sensitive ESR signal occurs at $\mathrm{g}=2.0018$ in fossil, but not modern enamel (Figure 1d; Tables 1, 2). Currently, most labs use placental mammal enamel, but marsupial and shark enamel also have datable signals (Blackwell et al., 2002b, 2004). Presumably, any vertebrate enamel should be datable, but this needs verification for each taxonomic order by extensive testing before general applicability can be assumed because tests with crocodile enamel showed $\mathrm{Fe}$ interference problems that hampered dating (Blackwell et al., unpublished data). Bones, dentine, some fish scales, and crustacean chitin also show the same signal (Figure 13) which grows similarly to that in tooth enamel. Rink et al. (2003) used the signal in authigenic apatite veins to date sequences in Tabun Cave, Israel, but non-organic apatites often lack radiation sensitive signals (Skinner et al., unpublished data). In tissues other than enamel, the signals do not fade, but their low sensitivity causes very low signal intensity unless the sample age approaches 0.8-1 Ma. Since diagenetic alteration in bone also complicates its use, bone dating has largely been abandoned in favour of enamel. Analyses for enameloid fish scales (e.g., gar, Lepisosteus) have been developed, but need further testing. In addition to interference problems, other fish scales do not appear to give sufficiently large signals for accurate dates. In HAP, ESR dates must consider $U$ uptake and ingrowth by $U$ daughters, as well as possible $\mathrm{Rn}$ loss and $\mathrm{U}$ leaching (Figure 7).

In HAP, the long ESR signal lifetime, $\tau \sim 10^{19} \mathrm{y}$ (Skinner et al., 2000), guarantees its utility. In mammals, its radiation-sensitivity does not depend on species, but does depend on the crystallinity which is affected by the animal's age and health (Skinner et al., 2001a). In deciduous teeth (i.e., "milk" teeth), poorly crystallized HAP causes analytical problems. Although signal saturation depends on the sample's $U$ concentration, saturation in enamel generally does not occur before the tooth is $\sim 5$ Ma. Teeth as old as 4.0 Ma have been dated successfully. Although some teeth as young as 8-10 ka have been dated, dosimetry experiments suggest that teeth with doses of 0.05-0.1 Gray may be datable (Wieser et al., 2005). Currently, few attempts have made to dates sites younger than $\sim 25-30 \mathrm{ka}\left(\sim 2-5\right.$ Gray), because ${ }^{14} \mathrm{C}$ dating is usually used instead.

The standard ESR method (i.e., not isochrons) for tooth enamel has now been tested extensively against other dating methods for sites in the age range 30-300 ka (Blackwell, 2001, Table 1), but for teeth $>300-400 \mathrm{ka}$, relatively few calibration tests have been attempted. Archaeological applications have been extensive. Despite calls for much more complex measurement protocols 


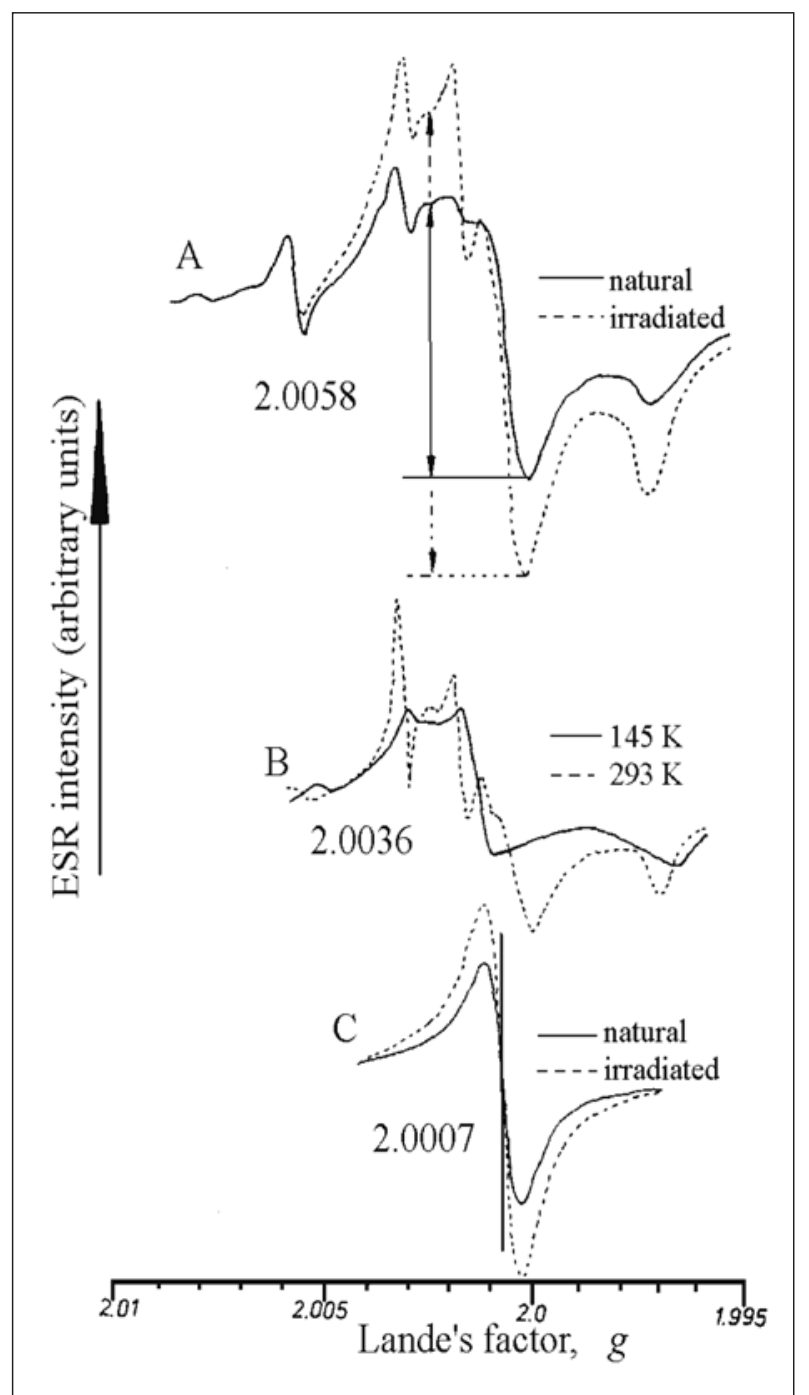

Fig. 11: ESR spectra in aragonitic mollusc shells.

Three signals commonly occur in aragonitic mollusc shells (adapted from Blackwell, 2001):

a. The signal at $g=2.0058$ before and after irradiation measured at room temperature.

$b$. The signal at $g=2.0036$ measured at room temperature $\left(293^{\circ} \mathrm{K}\right)$ and at $145^{\circ} \mathrm{K}$

c. The signal at $g=2.0007$ before and after irradiation measured at room temperature.

(e.g., Grün, 2002; Vanhaelewyn et al., 2000), Q band tests indicate that, although the peak is complex, it grows uniformly and can be accurately measured by a simple peak height measurement without deconvolution (Skinner et al., 2000). Human dosimetry experiments (Blackwell, 2001, Table 1) have hinted at possible problems with interference, temperature sensitivity, and signals induced by grinding and UV light exposure. Several researchers have suggested complex preparation techniques to com- pensate for these problems (e.g., Onori et al., 2000), but their effect on teeth older than 10 ka must be minimal or the ESR ages would not agree with those from other dating methods. While standard ESR can still be improved methodologically, such as by fully understanding U uptake, this does not hamper its application, especially in many caves, where the dental $U$ concentrations were so low that all the model ages are statistically identical (Figure 8a; e.g., Skinner et al., 2005).

For the isochron method in enamel, calibration tests have been completed against ${ }^{230} \mathrm{Th} /{ }^{234} \mathrm{U},{ }^{40} \mathrm{Ar} /{ }^{39} \mathrm{Ar}$, and standard ESR (e.g., Skinner et al., 2001b). Disagreements between standard ESR and isochrons imply changes in $\mathrm{D}_{\text {ext }}(\mathrm{t})$ or secondary $\mathrm{U}$ remobilization (Blackwell et al., 2001a, 2001b).

For enamel dating, molars and premolars from large herbivores make the best specimens, because both isochron and standard ESR analyses can be completed. Very small teeth are analyzed with the ramped dosing technique, but the enamel must be separated from the dentine manually. For small teeth, several teeth from the same jaw can be attempted for isochron analysis. ESR dating does not require that mammal teeth be fully identified, but other vertebrate groups have not been tested sufficiently to preclude taxonomic identification. Fragmentary teeth are fine, providing enough enamel and dentine remains for analysis (Table 1). For example, one mammoth molar plate provides enough enamel for an isochron. New non-destructive techniques using smaller teeth are being developed, but are not yet routine.

For bones, dentine, ivory (mixed dentine and enamel), and antler, the method is more difficult to apply and has not been particularly successful. Their low signal sensitivity causes, if nothing else, a much higher minimum age limit. For dentine, tests suggest that sensitivity problems can be overcome by using it to date teeth $>1 \mathrm{Ma}$ (Blackwell et al., 2002c). Diagenetic minerals in dentine cause few problems, except further lowering sensitivity (Skinner et al., 2000). In bone, tusk, and antler, contaminants and secondary mineralization can also complicate the signal measurement. Since all these tissues can absorb significant $\mathrm{U}$, uptake modelling becomes even more essential in determining accurate dates. Crustacean shell chitin shows a typical HAP signal, but the method needs development to determine if it might be applicable to brine shrimp or other chitinous species.

In caves, the applications have been too numerous to detail them all, but open-air karst applications have been more limited (Blackwell, 2001, Table 1). Dating at human paleontological and archaeological sites has been the most common use (e.g., Falguères, 2003), but nonhominid faunal applications (e.g., Godfrey-Smith et al., 2003) are becoming more common. ESR dates combined 


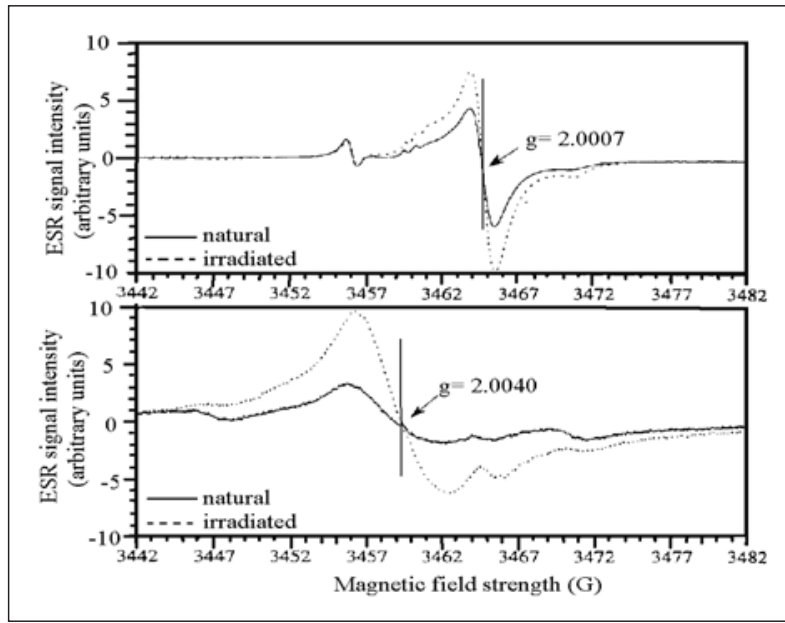

Fig. 12: ESR spectra in tufa and travertine.

In tufa and other slowly precipitated carbonates, the ESR spectra can vary dramatically, often due to interference signals from included organic matter, contaminant minerals, and trace elements (adapted from Blackwell, 2001).

with faunal, palynological, and geomorphological data at Treugol'naya Cave, in the Russian Caucasus, have begun to describe an extensive OIS 11 sequence (Doronichev et al., 2004). Blackwell et al. (2001b) used ESR isochrons to assess U uptake and ages for the hominid site at Bau de l'Aubesier.

At Divje babe I, Slovenia, a flute made from cave bear bone was found associated with Mousterian artefacts. Initially, Lau et al. (1997) showed the flute to be > $43 \mathrm{ka}$. Altogether, more than 40 subsamples were dated from 16 Ursus spelaeus (cave bear) teeth found in Layers 8 through 20 to build a detailed and precise chronostratigraphic sequence (Figure 14a) which allowed other sedimentological analyses to be tied to an absolute time sequence (e.g., Figure 14b; Turk et al., 2001). The resulting paleoclimatic interpretations were correlated with other global climatic events (e.g., Figure 14c; Turk et al., 2002).

\section{HEATED SILICA: VOLCANIC ASH, IGNEOUS ROCKS, BAKED SEDIMENT, BURNT FLINT AND CHERT}

Cave and karst sediment may preserve volcanic ash, tektites, and baked sediment, but few ESR applications have been attempted. Heated chert and flint artefacts occur in archaeological sites associated with Late Pleistocene and Holocene karst.

Quartz and silica exhibit several radiation-sensitive ESR signals (Figure 15). Due to the Ti and Ge signals' low sensitivity, fast saturation, and propensity for bleaching (e.g., Woda et al., 2001), most studies use the OHC, E', or Al signals. Some samples do require signal subtraction

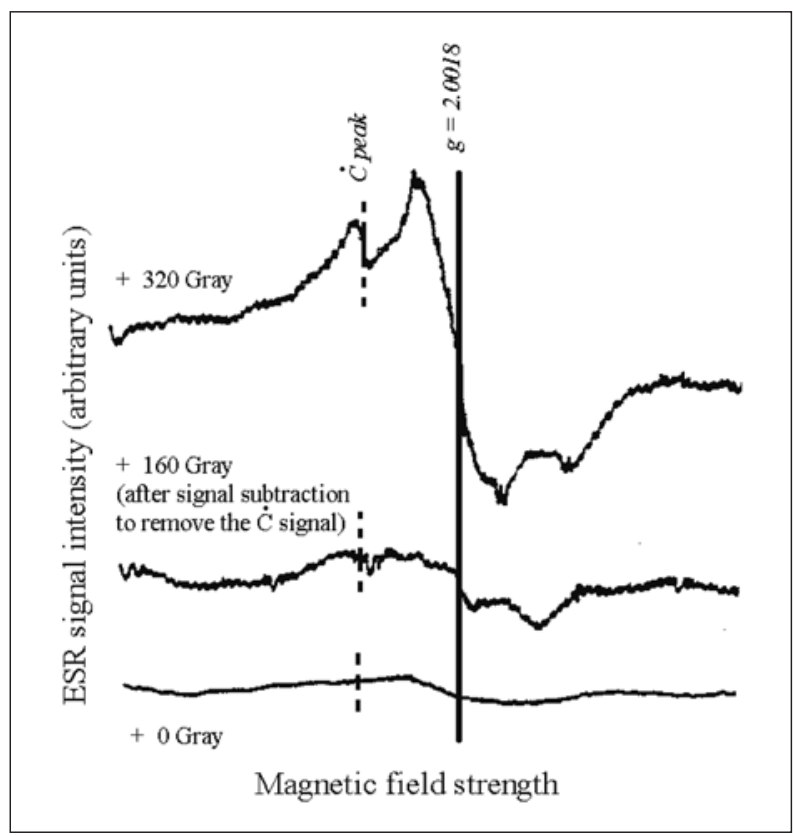

Fig. 13: The ESR hydroxyapatite signal in Lepisosteus platostomus (gar) scales.

In these scales from the Sangamonian lake at Hopwood Farm, $I L$, low signal intensity in the natural sample (lower) makes the signal difficult to discern, but artificial irradiation reveals the distinctive hydroxyapatite signal at $g=2.0018$, along with a carbon radical signal that partially interferes with the dating signal.

to remove trace contaminant interference signals (Figure $6 \mathrm{~b})$. Because quartz does not absorb U over time, its age calculations do not require modelling for $U$ uptake like tooth enamel. To provide meaningful dates, any preexisting geological signals, however, must have been zeroed completely during the depositional event (Figure 2b). In some flint, an unbleachable component may survive typical heating (Skinner, 2000). Signal lifetimes of $\tau \leq 100 \mathrm{y}$ were measured for the $E^{\prime}$ and $\mathrm{Al}$ signals, but heated flints show much longer lifetimes, suggesting that the signals' kinetics may change on heating. A short-lived interference signal, $E_{1}^{\prime}$, with $\tau=40 \mathrm{y}$, can interfere with $E^{\prime}$ signal measurement in some heated quartz samples (Toyoda, 2004), complicating dating for volcanic rocks and impact craters.

For burnt flint, chert, and quartz sand (Tables 1, 2), calibration tests against other methods and more basic studies are needed. The precision for $\mathrm{A}_{\Sigma}$ values from ESR compares well with those obtained from TL on the same materials. Flints and cherts as young as 10-20 ka may be datable, but the maximum dating limit, which depends on the flint type, has not yet been well established. Applications to dating burnt sand and volcanic ash are even less advanced, but theoretically feasible. Ulusoy (2003) and Beerten et al. (2003) have both been experimenting 
A

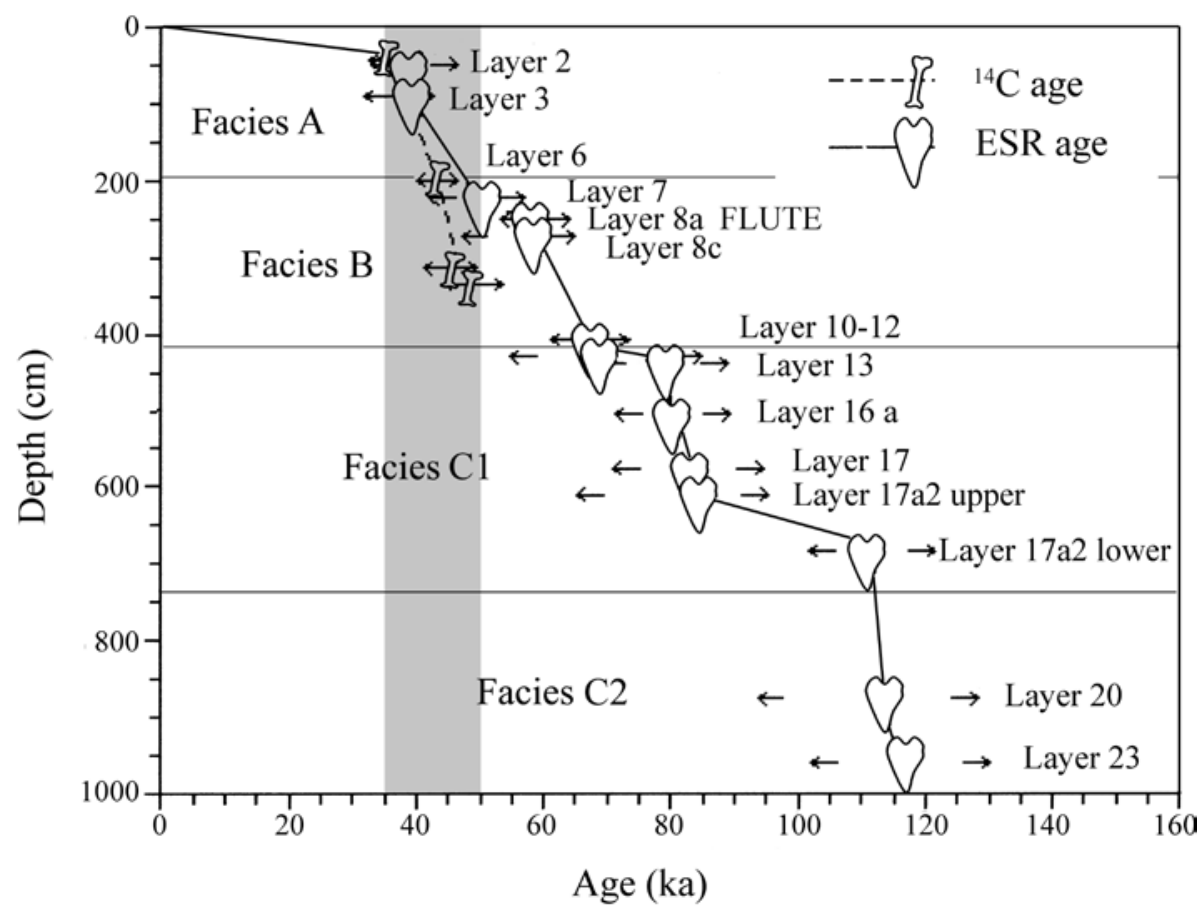

B

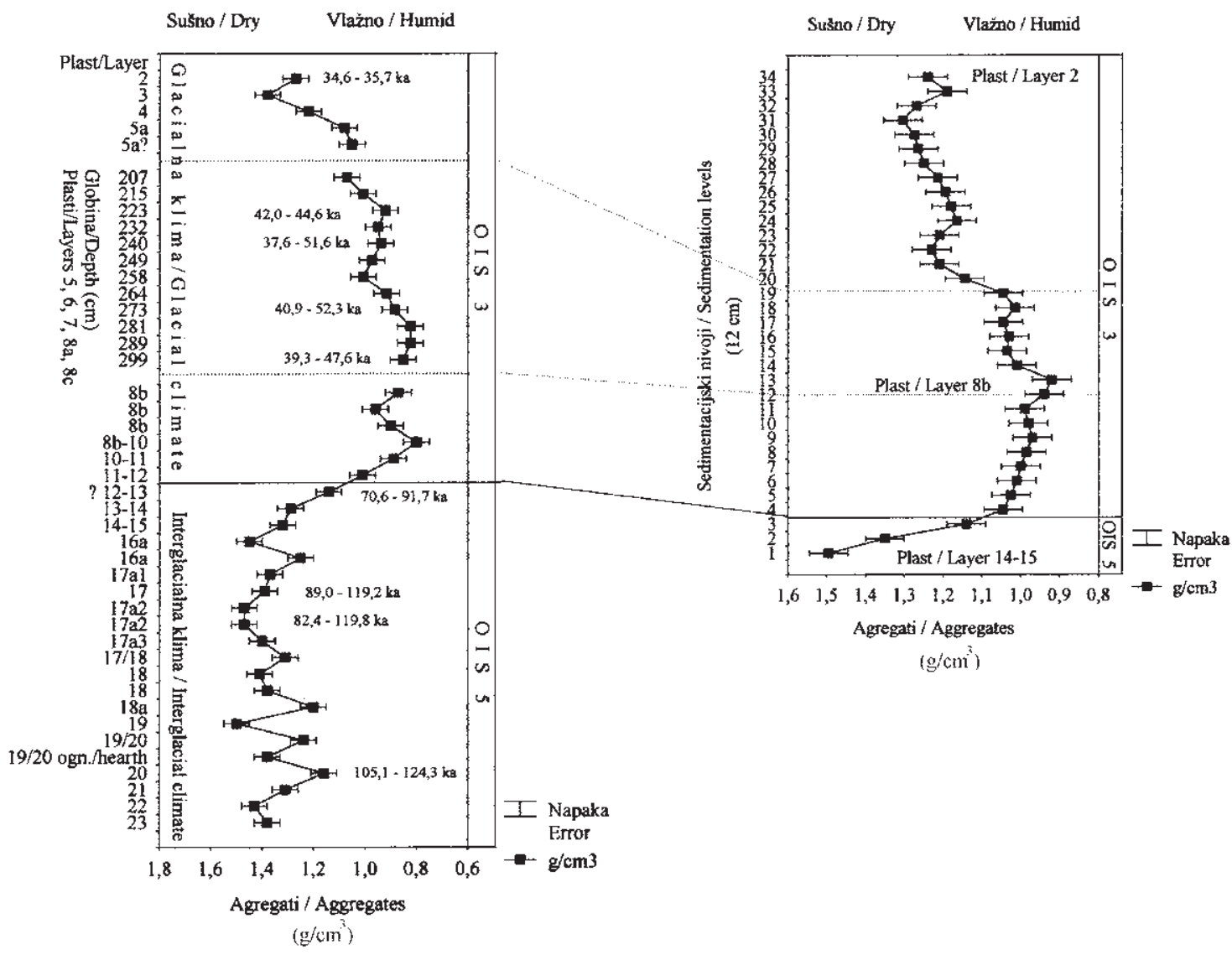

Figure 14: ESR dating at Divje babe I, Slovenia. 


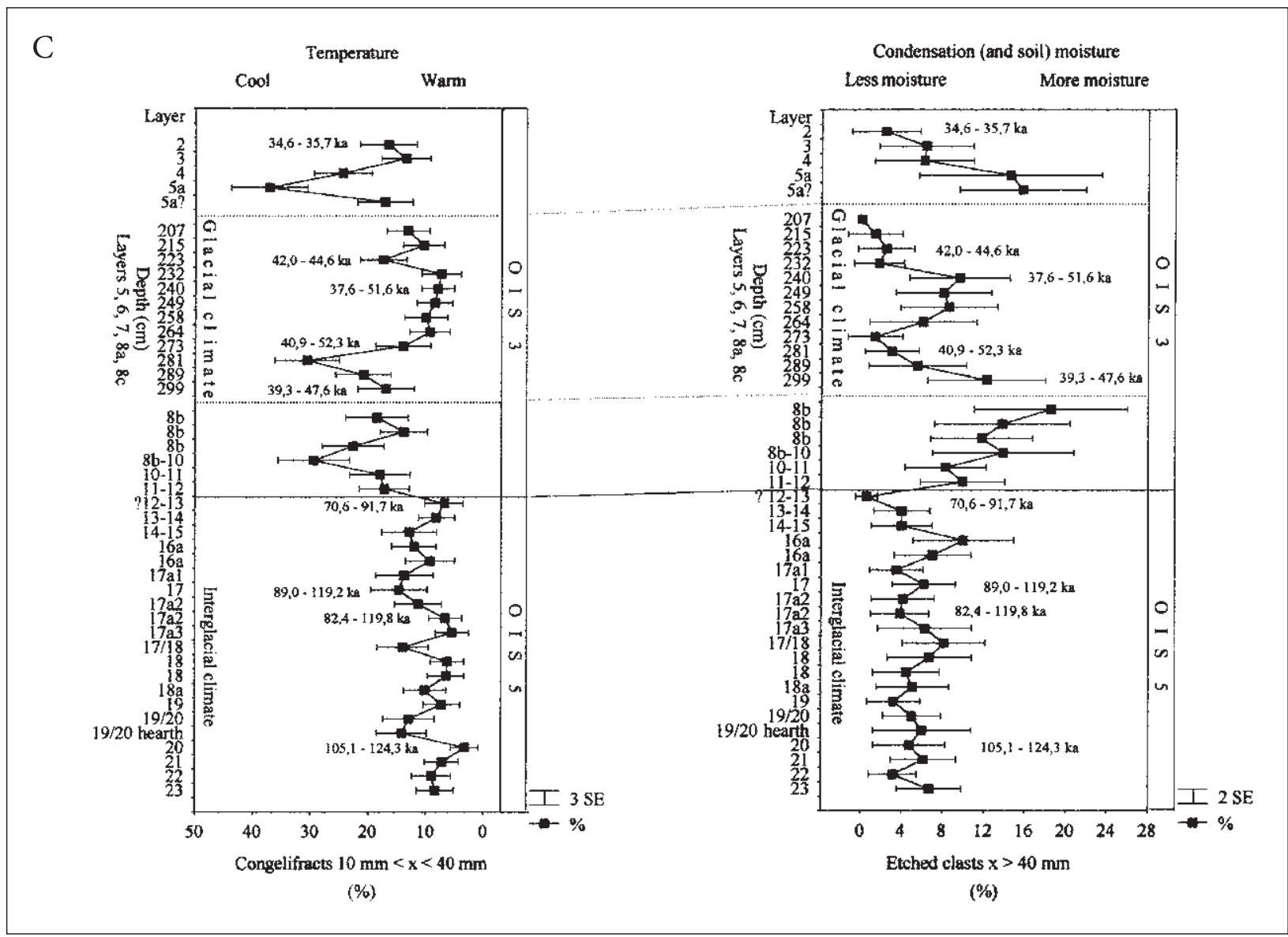

Fig. 14: ESR dating at Divje babe I, Slovenia.

Recently, a well dated sequence at Divje babe I cave, Slovenia, was coupled with detailed sedimentological analyses to develop detailed paleoclimatic interpretations and correlations with global climatic episodes:

A. The 16 teeth dated by ESR and four bones dated by ${ }^{14}$ C show that the cave filled in episodically, with depositional hiati at approximately 420 and $590 \mathrm{~cm}$ below datum.

B. Given the dates for the layers, aggregate analyses from the fine sediment fraction can be correlated with the global OIS curve (Turk et al., 2001).

C. The ESR, aggregate, and other sedimentary analyses combine to indicate paleoclimatic variations for the area around Divje babe I during the late Pleistocene (Turk et al., 2002).

with single crystal techniques for dating quartz. Tani et al. (1998) examined the thermal history for a flint artefact based on its ESR signals.

\section{STRAINED QUARTZ AND FELDSPAR, FAULT GOUGE, MYLONITE}

In many karst systems, caves develop along faults. ESR can date the most recent, and sometimes several earlier, fault movements (Figure 16), allowing complex tectonic histories to be unravelled. In Japan, the technique has been widely applied to numerous faults (Blackwell, 2001, Table 1), but few directly associated with caves. Tatumi et al. (2004) reported potentially datable signals in feldspar, while Mittani et al. (2004) tried using the $[\mathrm{Pb}-\mathrm{Pb}]^{3+}$ center in amazonite.
In dating gouge, strain zeroes the signals in the gouge minerals (Figure 3). Several grain sizes must be tested to ensure that the signals have been completely reset. Most researchers use the $E^{\prime}, \mathrm{OHC}$, or $\mathrm{Al}$ signals in quartz (Figure 15 ) or occasionally feldspar, but the grains must be selected by hand after heavy mineral separation and HF leaching to ensure that only gouge minerals with no secondary overgrowths are used. Lee and Schwarcz (2001) advocate using at least two signals to ensure accuracy.

\section{QUARTZ ZEROED BY LIGHT, BEACH SAND, LOESS, FLUVIAL SEDIMENT}

If a radiation-sensitive ESR signal found in quartz can be completely zeroed by exposure to strong light, as can 


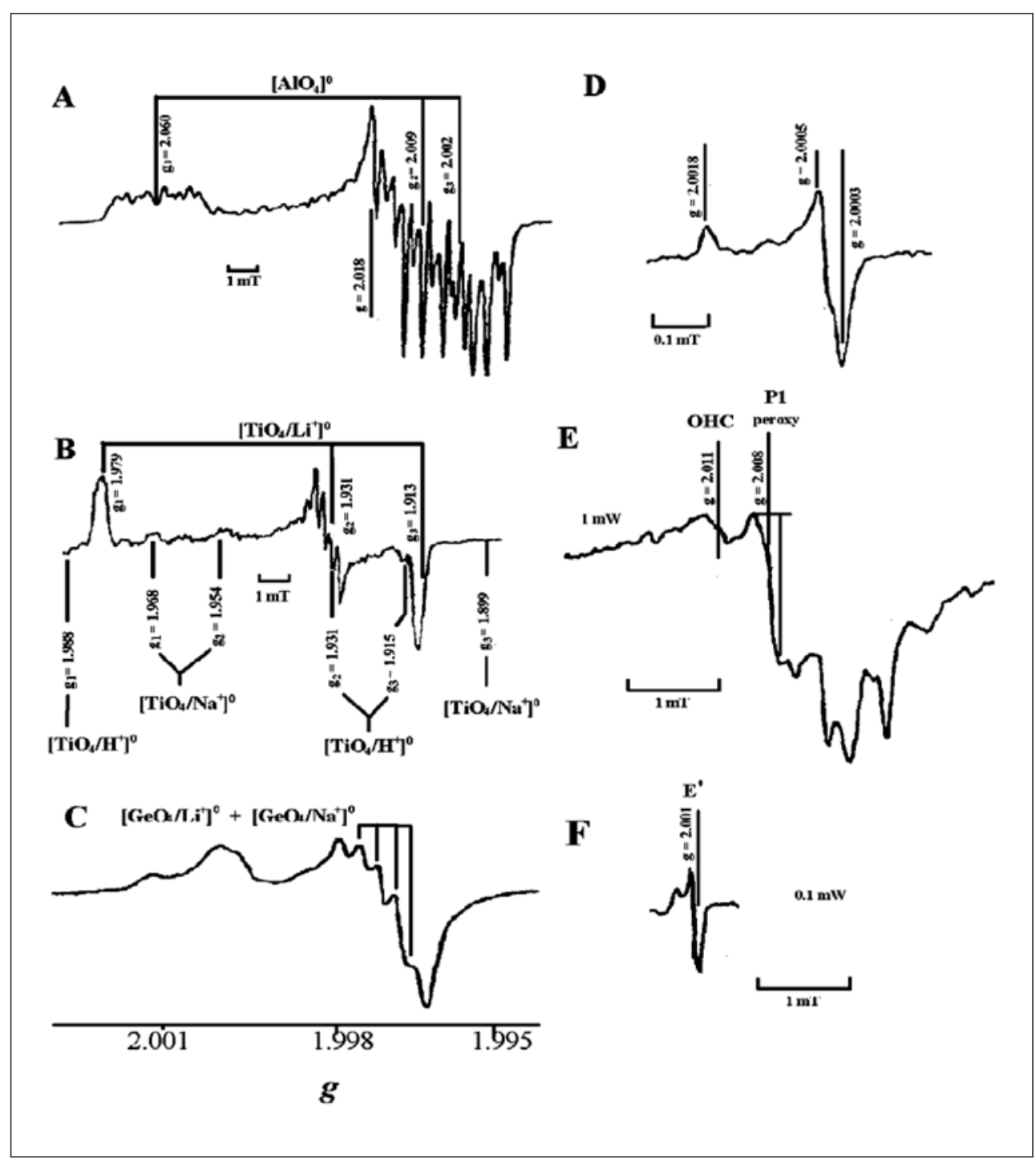

Fig. 15: ESR signals in quartz.

Several signals occur in quartz, flint, and fault gouge minerals (adapted from Blackwell, 2001):

a. The aluminum (Al) signal, often used for dating fault gouge, must be measured at $70^{\circ} \mathrm{K}$. It is an $\left(A l O_{4}\right)^{0}$ defect.

b. The titanium (Ti) signal, which has not been used often for dating arises from $\left(\mathrm{TiO}_{4} / \mathrm{H}^{+}\right)^{0},\left(\mathrm{TiO}_{4} / \mathrm{Li}^{+}\right)^{0},\left(\mathrm{TiO}_{4} / \mathrm{Na}^{+}\right)^{0}$ defects.

c. Because the germanium (Ge) signal is more easily bleached than most other signals in many quartz samples, it is used for dating quartz sediment. This complex signal arises from overlapping $\left(\mathrm{GeO}_{4} / \mathrm{Li}^{+}\right)^{0}$ and $\left(\mathrm{GeO}_{4} / \mathrm{Na}^{+}\right)^{0}$ defects.

d. The E' signal at $g=2.0001$ is easily measured at room temperature to date quartz, flint, and fault gouge.

e. The complex oxygen hole centre $(\mathrm{OHC})$ signal and the $\mathrm{P} 1$ (peroxy) signal are also measured at room temperature. OHC has been used to date quartz, flint, and fault gouge.

the Ge signal (Figure 2a), then its deposition in a shallow subaerial environment can be dated. As yet, it remains controversial whether any signal is completely zeroed during natural deposition (e.g., Toyoda et al., 2000; Voinchet et al., 2003). If sediment does not bleach completely, then any ages become maximum ages. Since most applications attempted thus far have used dubious analytical techniques (e.g., Blackwell, 2001, ), deciding if the results are fortuitous or genuine is difficult. Although these tech- niques await several basic theoretical studies, the recent successes with TL and OSL using similar sediment suggest that the potential exists here for many applications.

\section{AUTHIGENIC QUARTZ: PHYTOLITHS, DIATOMS, CEMENT, LATERITE, AND SILCRETE}

Both diatoms and phytoliths theoretically should be datable by ESR. Having a suitable signal, phytoliths need further investigation. Inherently, diatoms should also 


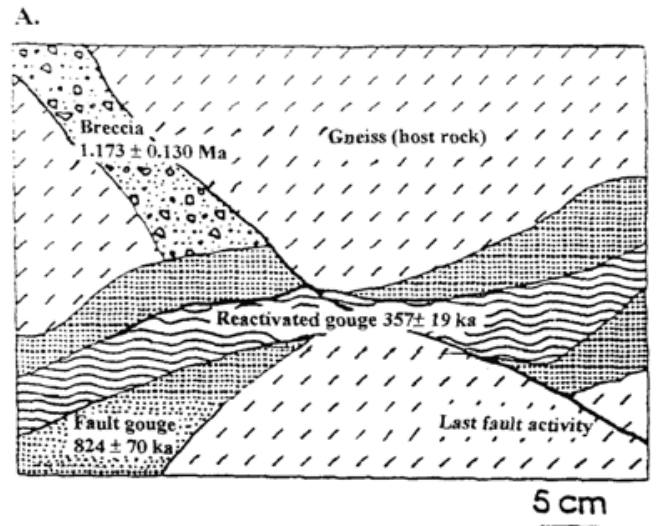

B.

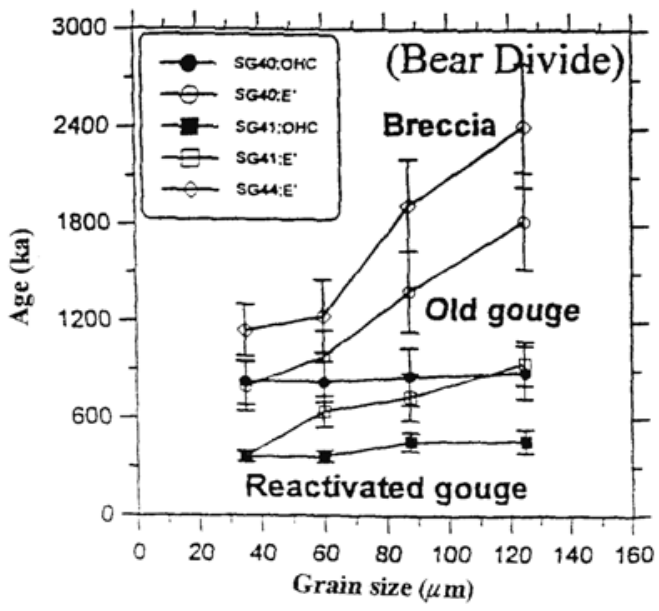

Fig. 16: Fault gouge dating.

In fault gouge from the Bear Divide, San Gabriel Fault, CA, the gouge records several periods of activity:

a. At least three earthquakes occurred in this outcrop at $357 \pm 19$, $824 \pm 70$, and $1173 \pm 130 \mathrm{ka}$.

b. Plotting the ESR ages vs. grain size shows different plateaux in old and reactivated fault gouge.

(after Lee \& Schwarcz, 1994).

have radiation sensitive signals similar to those in other quartz. In both, the ESR signals should be zero when the crystals form, thereby eliminating the problem of incomplete zeroing seen in other quartz applications.

Were one able to date laterite and silcrete, much geomorphic information might be discovered, but early attempts have not been systematically verified. Diagenetic alteration and secondary cementation may complicate these applications, creating complex curves. Nonetheless, all these have potential that should be developed further.

\section{CLAY MINERALS}

Several clay minerals have viable ESR signals. Both kaolinite and montmorillanite have an $\mathrm{OHC}$ signal associ-

ated with their silicate layers. In the latter, the stability, $\tau=10^{7}$ years at surface temperatures, suggests that its applicability for dating should include at least the Middle and Late Quaternary. Montmorillanite also has a radiation-sensitive carbonate signal, but with even lower stability. Radionuclides in associated Fe-oxides cause the signals in kaolinite, which have been used to fingerprint and source the clays. Fukuchi (2001) has tried using the OHC signal in montmorillanite to date Japanese faults. Bensimon et al. (2000) examined signal stabilities in natural clay signals. All these methods still need much development before routine application will be possible.

\section{OTHER SALTS: DOLOMITE, GYPSUM, GYPCRETE, HALITE, SULPHATES}

Dating salts can provide detailed information about associated karst features. Since salts frequently experience diagenesis, remineralization, and cementation, they require petrographic or geochemical checks to ensure accurate ages.

Several salts have strong ESR signals (Blackwell, 1995, Table 2). Strong radiation-sensitive signals in other carbonates, sulphates, and phosphates all show potential to be developed into viable techniques. Useful signals may also exist in rare salts with analogous geochemical formulae, but few have been examined. Success may hinge on the salts' purity, since the organic radicals, especially from humic acids, common in some subaerially precipitated salts tend to interfere with dating signals (e.g., Debuyst et al., 2000).

As yet, ESR dating has been attempted only for gypsum, anhydrite, halite, monohydrocalcite, dolomite, and barite, but not with unqualified success. Preliminary results on salt deposits indicate that signal intensities increase with sampling depth, but agreement with other dating methods has been poor. In gypsum, the $\mathrm{g}=2.0082$ signal gives the best results. Ulusoy (2004) studied gypsums from Turkey. Attempts to use gypcrete were hampered by the difficulties in obtaining sufficient sample for adequate growth curves to determine the $\beta$ efficiency factor, $\mathrm{k}_{\beta}$, which must be measured for each sample, due to differences in the precipitation history. Kohno et al. (1996) measured an accumulated dose in a barite desert rose. Once the idiosyncrasies in sample preparation have been standardized, these applications should provide interesting details about karst systems.

\section{OTHER APPLICATIONS}

Other applications include using ESR imaging systems to explore mineral (e.g., Gotze \& Plotze, 1997) and fossil growth and diagenesis (e.g., Tsukamoto \& Heikoop, 1996). Omura \& Ikeya (1995) used ESR microscopy to 
map gypsum crystal growth. Similar techniques could theoretically be applied to other salts. In a rather simplistic approach, Yugo et al. (1998) proposed a model for paleowind patterns based on the provenance of aeolian quartz dust as determined by the ESR intensity.

\section{CONCLUSIONS}

In caves, abris, and karst fissures, ESR dating has been particularly effective at dating teeth. While rare in caves, dating with molluscs, and other fossils also are easily applicable. Other methods have and are being developed that may prove extremely useful in future, including dating gypsum, dolomite, quartz, and other minerals.

In open-air karst settings, one must expect that changing sedimentary water concentrations, secondary leaching or addition of $U$ or Th in the sediment, and changing cosmic dose rates in response to burial will affect the external dose rates. Therefore, accurate dates must consider these phenomena carefully. While this complicates the age calculations, ESR can still provide accurate dates for many materials found associated with open-air karst environments, including teeth, egg shells, mollusc shells, burnt flint, fault gouge, and possibly for foraminifera, phytoliths, diatoms, and ostracodes.

\section{ACKNOWLEDGEMENTS}

Some examples cited herein were analyzed thanks to support from the National Science Foundation, the Leakey Foundation, Toyota Tapestry Foundation, RFK Science Research Institute, and Williams College. Over the years, Barry Brennan, Bill Prestwich, Jack Rink, Bill Buhay, Rainer Grün, Martin Jonas, Michel Barabas, Darren Curnoe, Eddie Rhodes, Ed Haskell, Anotoly Molod'kov, Albrecht Wieser, Ulrich Radtke, Galena Hütt, Neil Whitehead, Motoji Ikeya, Glen Berger, Anne Wintle,
Martin Aitken, Gerd Hennig, John Dennison, Andrew Pike, Christophe Falguères, Shin Toyoda, Mimi Divjak, Hee Kwon Lee, Daniel Richter, Hélène Valladas, Ruth Lyons, Naomi Porat, and especially Henry Schwarcz have provided valuable insights in discussions about ESR dating. Anne Skinner and Joel Blickstein provided many useful comments on this manuscript and assisted with its preparation. The reviewers provided excellent suggestions to improve the work.

\section{REFERENCES}

Bartoll, J., Stößer, R., \& Nofz, M., 2000: Generation and conversion of electronic defects in calcium carbonates by UV/Vis light. Applied Radiation \& Isotopes, 52, 1099-1105.

Beerten, K., Pierreux, D., \& Stesmans, A., 2003: Towards single grain ESR dating of sedimentary quartz: First results. Quaternary Science Reviews, 22, 13291334.

Bensimon, Y., Deroide, B., Dijoux, F., \& Martineau, M., 2000: Nature and thermal stability of paramagnetic defects in natural clay: A study by electron spin resonance. Journal of Physics and Chemistry of Solids, 61, 1623-1632.
Blackwell, B.A., 1994: Problems associated with reworked teeth in electron spin resonance dating. Quaternary Science Reviews (Quaternary Geochronology) 13, 651-660.

Blackwell, B.A., 1995: Electron spin resonance dating. In N.W. Rutter, N.R. Catto, eds. Dating Methods for Quaternary Deposits. Geological Association of Canada, St. John's, Geotext 2, 209-251.

Blackwell, B.A.B., 2001: Electron spin resonance (ESR) dating in lacustrine environments. In: W.M. Last, J.P. Smol, eds. Tracking Environmental Change Using Lake Sediments, Basin Analysis, Coring, and Chronological Techniques, vol. 1, 283-368, Kleuwer, Dordecht. 
Blackwell, B.A.B., \& Blickstein, J.I.B., 2000: Considering sedimentary $U$ uptake in external dose rates determinations for ESR and luminescent dating. Quaternary International, 68-71, 329-343.

Blackwell, B.A.B., Leung, H.Y.M., Skinner, A.R., Schwarcz, H.P., Lebel, S., Valladas, H., Blickstein, J.I.B. \& Divjak, M.N., 2000: External dose rate determinations for ESR dating at Bau de l'Aubesier, Provence. Quaternary International, 68-71, 345-361.

Blackwell, B.A.B., Skinner, A.R., \& Blickstein, J.I.B., 2001a: ESR isochron exercises: How accurately do modern dose rate measurements reflect paleodose rates? Quaternary Science Reviews (Quaternary Geochronology), 20, 1031-1039.

Blackwell, B.A.B., Skinner, A.R., Blickstein, J.I.B., Lebel, S., \& Leung, H.Y.M., 2001b: ESR isochron analyses at Bau de l'Aubesier, Provence: Clues to U uptake in fossil teeth. Geoarchaeology, 16, 719-761.

Blackwell, B.A.B., Skinner, A.R., Brassard, P., \& Blickstein, J.I.B., 2002a. U Uptake in tooth enamel: Lessons from isochron analyses and laboratory simulation experiments. In: Whitehead, N.E., Ikeya, M., eds., Proceedings of the International Symposium on New Prospects in ESR Dosimetry and Dating, Society of ESR Applied Metrology, Osaka, Advances in ESR Applications, 18, 97-118.

Blackwell, B.A.B., Chaderton, D.A. II, Skinner, A.R., Blickstein, J.I.B., Maisey, J.G., \& Divjak, M.N., 2002b: Developing ESR dating for sharks' teeth: Towards a new geolochronological method for sedimentological and paleontological analysis. Geological Society of America Abstracts with Program 34, A, Abstract 187-13.

Blackwell, B.A.B., Condiles, A.P., Skinner, A.R., \& Blickstein, J.I.B., 2002c: ESR dating of dentine: Triumphs and tribulations. Annual Meeting of the Paleoanthropology Society, Denver. Journal of Human Evolution, 42 (3), A7.

Blackwell, B.A.B., Nicholls, C., Teng, S., Tedford, R.E., Skinner, A.R., \& Blickstein, J.I.B., 2004: Developing ESR dating for marsupial teeth: Recognizing multiple sedimentation events in the Lake Eyre Basin, Australia. Geologicial Society of America Annual Meeting Program with Abstracts, 36, Abstract 227 13.

Blackwell, B.A.B., Liang, S.S., Lei, C.Y.Q.Q., Golovanova, L.V., Doronichev, V.B., Skinner, A.R., \& Blickstein, J.I.B., 2005a: ESR at Treugol'naya Cave, northern Caucasus Mt., Russia: Dating Russia’s oldest archaeological site and paleoclimatic change in Oxygen Isotope Stage 11. Applied Radiation \& Isotopes, 62, 237-245.
Blackwell, B.A.B., Montoya, A., Blickstein, J.I.B., Skinner, A.R., Pappu, S., Gunnell, Y., Taieb, M. \& Akhilesh, K., 2005b: ESR analyses the Paleolithic site, at Attirampakkam, India: Clues to U uptake, watertable migration, reworking, and paleoenvironmental change. 2005 Luminescent \& ESR Dating Conference, /Köln

Brennan, B.J., 2000: Systematic underestimation of the age of samples with saturating exponential behaviour and inhomogeneous dose distribution. Radiation Measurements, 32, 731-734.

Brennan, B.J., Prestwich, W.V., Rink, W.J., Marsh, R.E., \& Schwarcz, H.P., 2000: a and b dose gradients in tooth enamel. Radiation Measurements, 32, 759-765.

Debuyst, R., Frenchen, M., \& Idrissi, S., 2000: Problems encountered in a TL and ESR study of natural monohydrocalcite. Radiation Measurements, 32, 725-729.

Doronichev, V.B., Blackwell, B.A.B., Golovanova, L.V., Levkovskaya, G.M., \& Pospelova, G.A., 2004: Treugol'naya Cave in the northern Caucasus, Russia: Its chronology, paleoenvironments, industries, and Relationship to the Lower Paleolithic in Eastern Europe. European Prehistory, 2, 77-144.

Eggins, S., Grün, R., Pike, A.W.G., Shelley, M., \& Taylor, L., 2003: 238U, 232Th profiling, and U-series isotope analysis of fossil teeth by laser-ablation ICPMS. Quaternary Science Reviews, 22, 1373-1382.

Falguères, C., 2003: ESR dating and the human evolution: Contributions to the chronology of the earliest humans in Europe. Quaternary Science Reviews, 22, 1345-1351.

Fukuchi, T., 2001: Assessment of fault activity by ESR dating of fault gouge; an example of the $500 \mathrm{~m}$ core samples drilled into the Nojima earthquake fault in Japan. Quaternary Science Reviews, 20, 1005-1008.

Godfrey-Smith, D.I., Grist, A.M., \& Stea, R.R., 2003: Dosimetric and radiocarbon chronology of a pre-Wisconsinan mastodon fossil locality at East Milford, Nova Scotia, Canada. Quaternary Science Reviews, 22, 1353-1360.

Gotze, J., \& Plotze, M., 1997: Investigation of trace element distribution in detrital quartz by electron paramagnetic resonance (EPR). European Journal of Mineralogy 9, 529-537.

Grün, R., 2002: ESR dose estimation on fossil tooth enamel by fitting the natural spectrum into the irradiated spectra. Radiation Measurements, 35, 87-93.

Grün, R., Kohno, H., Tani, A., Yamanaka, C., Ikeya, M., \& Huang, H.P., 1997: Pulsed ESR measurements on fossil teeth. Radiation Measurements 27, 425-432. 
Guibert, P., Bechtel, G., Schvoerer, M., Müller, P., \& Balescu, S., 1998. A new method for c dose rate estimation of heterogeneous media in TL dating. Radiation Measurements, 29, 663-671.

Hoffmann, D., \& Mangini, A., 2003: A method for coupled ESR/U-series dating of teeth showing postdepositional U-loss. Quaternary Science Reviews, 22, 1367-1372.

Ikeya, M., Ochiai, H., \& Tani, A., 1997: Total dose (Td) formula for uranium saturation-uptake model for ESR and TL dating. Radiation Measurements, 27, 339-343.

Kinoshita, A., Karmann, I., da Cruz, F.W. Jr., \& Baffa, O., 2004: K-band ESR spectra of calcite stalagmites from southeast and south Brazil. Applied Radiation \& Isotopes.

Kohno, H., Yamanaka, C., \& Ikeya, M., 1996: Effects of $\alpha$ irradiation and pulsed ESR measurements of evaporites. Applied Radiation \& Isotopes, 47, 1459-1463.

Lau, B., Blackwell, B.A.B., Schwarcz, H.P., Turk, I., \& Blickstein, J.I.B., 1997: Dating a flautist? Using ESR (electron spin resonance) in the Mousterian cave deposits at Divje Babe I, Slovenia. Geoarchaeology, 12, 507-536.

Lee, H.K., \& Schwarcz, H.P., 1993: An experimental study of shear-induced zeroing of ESR signals in quartz. Applied Radiation \& Isotopes, 44, 191-195.

Lee, H.K., \& Schwarcz, H.P., 1994: Criteria for complete zeroing of ESR signals during faulting of the San Gabriel Fault Zone, Southern California. Tectonophysics, 235, 317-337.

Lee, H.K., \& Schwarcz, H.P., 2001: ESR dating of the subsidiary faults in the Yangsan fault system, Korea. Quaternary Science Reviews, 20, 999-1003.

Lee, H.K., Rink, W.J., \& Schwarcz, H.P., 1997: Comparison of ESR signal dose-responses in modern and fossil tooth enamels. Radiation Measurements, 27, 405-411.

Lyons, R.G., \& Tan, S.M., 2000: Differentials or integrals: Pluses and minuses in their application to additive dose techniques. Applied Radiation \& Isotopes, 52, 1051-1057.

Miki, T., Murata, T., Kumai, H., \& Yamashiro, A., 1996: A high resolution EPR-CT microscope using cavityresonators equipped with small field gradient coils. Applied Radiation \& Isotopes, 47, 1599-1603.

Mittani, J.C.R., Cano, N.F., \& Watanabe, S., 2004: Use of the $[\mathrm{Pb}-\mathrm{Pb}] 3+$ center in amazonite for dating. Applied Radiation \& Isotopes.

Miyake, M., Liu, K.J., Walczak, T.M., \& Swartz, H.M., 2000: In vivo dosimetry of accidental exposures to radiation: Experimental results indicating the feasibility of practical use in human subjects. Applied Radiation \& Isotopes, 52, 1031-1038.
Mizuta, Y., Iida, S., Iima, Y., Ikeya, M., \& Yamanaka, C., 2002: TE111 ESR cavity with an aperature for scanning ESR imaging. In: Whitehead, N.E., Ikeya, M., eds., Proceedings of the International Symposium on New Prospects in ESR Dosimetry and Dating, Society of ESR Applied Metrology, Osaka, Advances in ESR Applications, 18, 299-302.

Molod'kov, A.N., 2001: ESR dating for early man at a Lower Palaeolithic cave-site (sic) in the Northern Caucasus as derived from terrestrial mollusc shells. Quaternary Science Reviews, 20, 1051-1055.

Nagy, V., 2000: Accuracy considerations in EPR dosimetry. Applied Radiation \& Isotopes, 52, 1039-1050 .

Oka, T., Ikeya, M., Sugawara, N., \& Nakanishi, A., 1996: A high-sensitivity portable spectrometer for ESR dosimetry. Applied Radiation \& Isotopes, 47, 1589 1594.

Oka, T., Grün, R., Tani, A., Yamanaka, C., Ikeya, M., \& Huang, H.P., 1997: ESR microscopy of fossil teeth. Radiation Measurements, 27, 331-337.

Olley, J.M., Roberts, R.G. \& Murray, A.S., 1997: Disequilibria in the uranium series decays series in the sedimentary deposits at Allen's Cave, Nullabor Plain, Australia: Implications for dose rate determinations. Radiation Measurements, 27, 433-443.

Omura, T., \& Ikeya, M., 1995: Evaluation of the ambient environment of mineral gypsum (CaS04.H20) growth by ESR microscope. Geochemical Journal, 29, 317-324.

Onori, S., Aragno, D., Fattibene, P., Petetti, E., \& Pressello, M.C., 2000: ISS protocol for EPR tooth enamel dosimetry. Radiation Measurements, 32, 787-792.

Pike, A.W.G., \& Hedges, R.E.M., 2001: Sample geometry and $U$ uptake in archaeological teeth, implications for U-series and ESR dating. Quaternary Science Reviews, 20, 1021-1025

Rink, W.J., Bartoll, J., Goldberg, P., \& Ronen, A., 2003: ESR dating of archaeologically relevant authigenic terrestrial apatite veins from Tabun Cave, Israel. Journal of Archaeological Science, 30, 1127-1138.

Robertson, S., \& Grün, R., 2000: Dose determination on tooth fragments from two human fossils. Radiation Measurements, 32, 773-779.

Schauer, D.A., Desrosiers, M.F., Kuppusamy, P., \& Zweier, J.L., 1996: Radiation dosimetry of an accidental overexposure using EPR spectrometry and imaging of human bone. Applied Radiation \& Isotopes, 47, 1345-1350.

Schellmann, G., \& Radtke, U., 2001: Progress in ESR dating of Pleistocene corals: A new approach for DE determination. Quaternary Science Reviews, 20, 1015-1020. 
Shih, T.S., Sato, H., Ikeya, M., Liew, P.M., \& Chien, S.H., 2002. Conditions and new extrapolation method for ESR dating of aragonitic mollusk (sic) shells. In: Whitehead, N.E., Ikeya, M., eds., Proceedings of the International Symposium on New Prospects in ESR Dosimetry and Dating, Society of ESR Applied Metrology, Osaka, Advances in ESR Applications, 18, 31-39.

Skinner, A.R., 2000: ESR dating: Is it still an 'experimental' technique? Applied Radiation \& Isotopes, 52, 1311-1316.

Skinner, A.R., Blackwell, B.A.B., Chasteen, D.E., Shao, J.M., \& Min, S.S., 2000: Improvements in dating tooth enamel by ESR. Applied Radiation \& Isotopes, 52, 1337-1344.

Skinner, A.R., Blackwell, B.A.B., Chasteen, D.E., \& Shao, J.M., 2001a: Q band ESR studies of fossil tooth enamel. Quaternary Science Reviews (Quaternary Geochronology), 20, 1027-1030.

Skinner, A.R., Blackwell, B.A.B., \& Lothian, V., 2001b: Calibrating ESR ages in the 2-Ma range at Olduvai Gorge, Tanzania. Paleoanthropology Society, Kansas City, MO. Journal of Human Evolution, 40 (3), A22.

Skinner, A.R., Blackwell, B.A.B., Martin, S.A., Ortega, A.J., Blickstein, J.I.B., Golovanova, L.V., \& Doronichev, V.B., 2005: ESR dating at Mezmaiskaya Cave, Russia. Applied Radiation \& Isotopes, 62, 219-224.

Tani, A., Bartoll, J., Ikeya, M., Komura, K., Kajiwara, H., Fujimura, S., Kamada, T., \& Yokoyama, Y., 1998: ESR study of thermal history and dating of a stone tool. Applied Magnetic Resonance, 13, 561-569.

Toyoda, S., 2004: Formation and decay of the $\mathrm{E}_{1}^{\prime}$ center and its precursor in natural quartz: Basics and applications. Applied Radiation \& Isotopes.

Toyoda, S., Voinchet, P., Falguères, C., Dolo, J.M., \& Laurent, M., 2000: Bleaching of ESR signals by the sunlight, a laboratory experiment for establishing the ESR dating of sediments. Applied Radiation \& Isotopes, 52, 1357-1362.

Tsukamoto, Y., \& Heikoop, J., 1996: Sulfite radicals in banded coral. Applied Radiation \& Isotopes, 47, 1437-1441.

Turk, I., Blackwell, B.A.B., Sabine, J., \& Dirjec, J., 2001: Morphometric and chronostratigraphic sedimentary analyses and paleoclimatic interpretations for the profile at Divje babe I, Slovenia. Arheološki vestnik, 52, 223-249.

Turk, I., Skaberne, D., Dirjec, J., \& Blackwell, B.A.B., 2002: Assessing humidity in an Upper Pleistocene karst environment: Palaeoclimates and palaeomicroclimates at the cave, Divje babe I, Slovenia. Acta carsologica, 31, 139-175.
Ulusoy, Ü.L.K., 2003: ESR dating of North Anatolian (Turkey) and Nojima (Japan) faults. Quaternary Science Reviews, 23, 161-174.

Ulusoy, Ü.L.K., 2004: ESR studies of Anatolian gypsum. Spectrochimica Acta A, Molecular \& Biomolecular Spectroscopy, 60, 1359-1365.

Vanhaelewyn, G., Callens, F., \& Grün, R., 2000: ESR spectrum deconvolution and dose assessment of fossil tooth enamel using maximum likelihood common factor analysis. Applied Radiation \& Isotopes, 52, 1317-1326.

Voinchet, P., Falguères, C., Laurent, M., Toyoda, S., Bahain, J.J., \& Dolo, J.M. 2003: Artificial optical bleaching of the aluminium center in quartz implications to ESR dating of sediments. Quaternary Science Reviews, 22, 1335-1338.

Whitehead, N.E., Lyon, G.L., Claridge, G.C., Sato, H. \& Ikeya, M., 2002: ESR studies on Antarctic carbonates and sulphates. In: Whitehead, N.E., Ikeya, M., eds., Proceedings of the International Symposium on New Prospects in ESR Dosimetry and Dating, Society of ESR Applied Metrology, Osaka, Advances in ESR Applications, 18, 11-18.

Wieser, A., Debuyst, R., Fattibene, P., Meghzifene, A., Onori, S., Bayankin, S.N., Blackwell, B.A.B., Brik, A., Bugay, A., Chumak, V., Ciesielski, B., Hoshi, H., Imata, H., Ivannikov, A., Ivanov, D., Junczewska, M., Miyazama, C., Pass, B., Penkowski, M., Pivovarov, S., Romanyukha, A., Romanyukha, L., Schauer, D., Scherbina, O., Schultka, K., Shames, A., Sholom, S., Skinner, A.R., Skvortsov, V., Stepanenko, V., Tielewuhan, E., Toyoda, S., \& Trompier, F., 2005: The 3rd international intercomparison on EPR tooth dosimetry: Part 1, general analysis. Applied Radiation \& Isotopes, 62, 163-171.

Woda, C., Mangini, A., \& Wagner, G.A., 2001: ESR dating of xenolithic quartz in volcanic rocks. Quaternary Science Reviews, 20, 993-998.

Yugo, O., Naruse, T., Ikeya, M., Kohno, H., \& Toyoda, S., 1998: Origin and derived courses of eolian dust quartz deposited during Marine Isotope Stage 2 in east Asia suggested by ESR signal intensity. Global \& Planetary Change, 18, 129-135. 
